\title{
Analysis of Cellular and Cell-Free Massive MIMO with Rician Fading Özgecan Özdogan
}




\title{
Analysis of Cellular and Cell-Free Massive MIMO with Rician Fading
}

\author{
Özgecan Özdogan
}

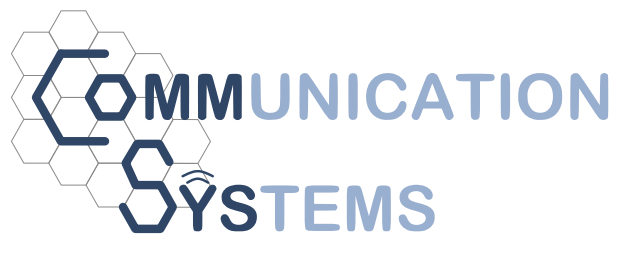

Division of Communication Systems Department of Electrical Engineering (ISY)

Linköping University, 58183 Linköping, Sweden

www.commsys.isy.liu.se

Linköping 2020 
This is a Swedish Licentiate Thesis.

The Licentiate degree comprises 120 ECTS credits of postgraduate studies.

Analysis of Cellular and Cell-Free Massive MIMO with Rician Fading

(C) 2020 Özgecan Özdogan, unless otherwise noted.

ISBN 978-91-7929-911-8

ISSN 0280-7971

Printed in Sweden by LiU-Tryck, Linköping 2020 


\section{Abstract}

The data traffic in cellular networks has grown at an exponential pace for decades. This trend will most probably continue in the future, driven by new innovative applications. One of the key enablers of future cellular networks is the massive MIMO technology. A massive MIMO base station is equipped with a massive number (e.g., a hundred) of individually steerable antennas, which can be effectively used to serve tens of user equipments simultaneously on the same time-frequency resource. It can provide a notable enhancement of both spectral efficiency and energy efficiency in comparison with conventional MIMO.

In the literature, the achievable spectral efficiencies of massive MIMO systems with a practical number of antennas have been rigorously characterized and optimized when the channels are subject to either spatially uncorrelated or correlated Rayleigh fading. Typically, in massive MIMO research, i.i.d. Rayleigh fading or less frequently free-space line-of-sight (LoS) channel models are assumed since they simplify the analysis. Massive MIMO technology is able to support both rich scattering and LoS scenarios. However, practical channels can consist of a combination of an LoS path and a correlated small-scale fading component caused by a finite number of scattering clusters that can be modeled by spatially correlated Rician fading. In the first part of this thesis, we consider a multi-cell scenario with spatially correlated Rician fading channels and derive closed-form achievable spectral efficiency expressions for different signal processing techniques.

Alternatively, a massive number of antennas can be spread over a large geographical area and this concept is called cell-free massive MIMO. In the canonical form of cell-free massive MIMO, the access points cooperate via a fronthaul network to spatially multiplex the users on the same time-frequency resource using network MIMO methods that only require locally obtained channel state information. Cellfree massive MIMO is a densely deployed system. Hence, the probability of having an LoS path between some access points and the users is quite high. In the second part of this thesis, we consider a practical scenario where the channels between the access points and the users are modeled with Rician fading. 


\section{Populärvetenskaplig sammanfattning}

Datatrafiken i mobilnät har vuxit exponentiellt i årtionden. Trenden kommer troligen att fortsätta i framtiden, drivet av nya innovativa applikationer. En av de viktigaste möjliggörarna för framtida mobilnät är massiv MIMO-teknik. En massiv MIMObasstation är utrustad med ett massivt antal (t.ex. hundratals) individuellt styrbara antenner, som effektivt kan användas för att betjäna tiotals användarenheter samtidigt på samma tids- och frekvensresurs. Det kan ge en stor förbättring av både spektraleffiktivitet och energieffektivitet jämfört med konventionell MIMO. Alternativt kan ett massivt antal antenner spridas över ett stort geografiskt område och detta koncept kallas cellfri massiv MIMO.

I både cellulär och cellfri massiv MIMO anländer den trådlösa signalen till mottagaren genom flera olika vägar. Typiskt kan en av vägarna vara siktlinje (LoS) och andra kan orsakas av ett begränsat antal spridande objekt i miljön. Denna typ av kommunikationskanal kan modelleras med rumsligt korrelerad Riceisk fädning. I massiv MIMO-litteraturen ignoreras LoS-vägen vanligtvis och antalet spridare antas vara oändligt eftersom dessa antaganden förenklar analysen. I den här avhandlingen antar vi rumsligt korrelerade Riceiskt fädande kanaler och härleder uppnåeliga spektraleffektivitetsuttryck i sluten form för olika signalbehandlingstekniker för både cellulär och cellfri massiv MIMO. 


\section{Acknowledgments}

First and foremost, I would like to express my deep gratitude and profound respect to my Ph.D. supervisor Dr. Emil Björnson for always finding exciting research directions and giving me freedom and encouragement. He always guided me with his helpful comments and advice whenever I feel stuck. From the very first day, his patience, support, enthusiasm, and immense knowledge have inspired and motivated me. Likewise, I would like to thank my co-supervisor Dr. Erik G. Larsson, for his expert advice, insightful comments, and suggestions.

My sincere thanks go to Dr. Jiayi Zhang (Beijing Jiaotong University) for providing expert feedback and productive collaborations.

The corridor of communication systems has provided a warm and friendly environment, thanks to all past and present colleagues. I have learned a lot from them during the time we have spent together. Specifically, I am thankful to my office mate Giovanni Interdonato for his strong support.

I want to thank my parents and my sister for their never-ending support and unconditional love. Besides, I would also like to thank my second family, my husband's family, for their support that makes tough times a lot easier. Last but not least, my heartfelt thanks go to my husband Vedat, who keeps me grounded.

Özgecan Özdogan Linköping, January 2020 


\section{Contents}

1 Introduction 1

1.1 Background and Motivation _............... 1

1.2 Channel Models for Massive MIMO . . . . . . . . . . . . . . 3

1.2.1 Channel Fading . . . . . . . . . . . . . . . . . . 4

1.2.2 Line-of-Sight Channels . . . . . . . . . . . . . . . 6

1.2.3 Spatially Uncorrelated Rayleigh Fading . . . . . . . . 9

1.2.4 Spatially Correlated Rayleigh Fading . . . . . . . . . . . 11

1.2.5 Spatially Correlated Rician Fading . . . . . . . . . . . . 16

1.2.6 Impact of LoS path and Spatial Correlation in Massive MIMO 18

2 Contributions of the Thesis 23

2.1 Papers Included in the Thesis . . . . . . . . . . . . . . 23

2.2 Papers Not Included in the Thesis . . . . . . . . . . . 25

$\begin{array}{ll}\text { Bibliography } & 27\end{array}$

$\begin{array}{ll}\text { Included Papers } & 35\end{array}$

A Massive MIMO with Spatially Correlated Rician Fading Channels 37

1 Introduction . . . . . . . . . . . . . . . . . . 39

1.1 Main Contributions . . . . . . . . . . . . . . . 40

2 Channel and System Model . . . . . . . . . . . . . . . . . 41

3 Channel Estimation . . . . . . . . . . . . . . . . . 42

3.1 MMSE Channel Estimator . . . . . . . . . . . . . . 43

3.2 Element-wise MMSE Channel Estimator . . . . . . . . . 44

3.3 LS Channel Estimator . . . . . . . . . . . . . . . . 44

4 Uplink Spectral efficiency with MR combining . . . . . . . . . 45

$4.1 \quad$ Uplink Spectral Efficiency with MMSE estimator . . . . . 46

4.2 Uplink Spectral Efficiency with EW-MMSE Estimator . . 47

4.3 Uplink Spectral Efficiency with LS Estimator . . . . . . . 48 
4.4 Uplink Spectral Efficiency with Mean Only Estimator . . . 49

5 Downlink Spectral Efficiency with MR Precoding . . . . . . . . 50

$5.1 \quad$ Downlink Spectral Efficiency with MMSE Estimator . . . 50

5.2 Downlink Spectral Efficiency with EW-MMSE Estimator . 52

5.3 Downlink Spectral Efficiency with LS Estimator . . . . . 52

5.4 Downlink Spectral Efficiency with Mean Only Estimator . 53

6 Asymptotic Analysis . . . . . . . . . . . . . . 53

6.1 Asymptotic Analysis of Spectral Efficiency with MMSE Estimator . . . . . . . . . . . . . . . 54

6.2 Asymptotic Analysis of Spectral Efficiency with EW-MMSE Estimator . . . . . . . . . . . . . . 55

6.3 Asymptotic Analysis of Spectral Efficiency with LS Estimator 56

6.4 Asymptotic Analysis of Spectral Efficiency with Mean Only Estimator . . . . . . . . . . . . . . 56

6.5 Discussion on the Asymptotic Behaviors of Rician Fading Channels . . . . . . . . . . . . . 57

$7 \quad$ Numerical Results . . . . . . . . . . . . . . . . . . . . . . 57

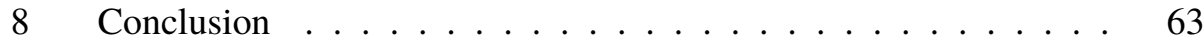

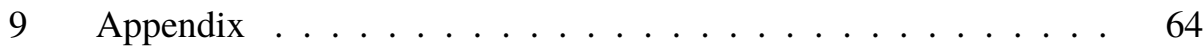

9.1 Useful results . . . . . . . . . . . . . . . . . . 64

9.2 Proof of Lemma 4 and Lemma $5 \ldots \ldots$. . . . . . . 65

9.3 Proof of Lemma $3 \ldots \ldots \ldots 6$

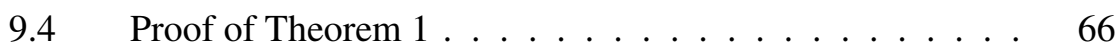

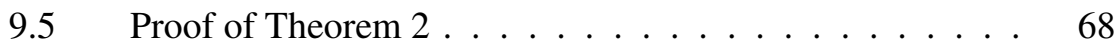

9.6 Proof of Theorem $3 \ldots \ldots \ldots \ldots$

9.7 Proof of Theorem 7 and Theorem $8 \ldots \ldots \ldots$

9.8 Proof of Theorem 9 and Theorem $10 \ldots \ldots$. . . . 70

9.9 Proof of Theorem $11 \ldots \ldots \ldots 71$

References . . . . . . . . . . . . . . . . . 72

B Cell-Free Massive MIMO with Rician Fading and Phase Shifts $\quad 77$

1 Introduction . . . . . . . . . . . . . . . . . . 79

2 System Model . . . . . . . . . . . . . . . . . . . . 81

3 Uplink Channel Estimation . . . . . . . . . . . . . . . . . 82

3.1 Phase-aware MMSE Channel Estimator . . . . . . . . . 83

$3.2 \quad$ LMMSE Channel Estimator . . . . . . . . . . . . . . 84

3.3 LS Channel Estimator with No Prior Information . . . . . 85

4 Uplink Data Transmission . . . . . . . . . . . . . . 86

4.1 Uplink Spectral Efficiency with the Phase-aware MMSE Estimator . . . . . . . . . . . . . . . . 89 
4.2 Uplink Spectral Efficiency with the LMMSE Estimator . 89

4.3 Uplink Spectral Efficiency with the LS Estimator . . . . . 90

5 Coherent Downlink Transmission ............. 91

5.1 Coherent Downlink Spectral Efficiency with the Phase-aware MMSE Estimator . . . . . . . . . . . . . . . . . 92

5.2 Coherent Downlink Spectral Efficiency with the LMMSE Estimator . . . . . . . . . . . . . . . 93

5.3 Coherent Downlink Spectral Efficiency with the LS Estimator 94

6 Non-coherent Downlink Transmission . . . . . . . . . . . . 94

6.1 Non-coherent Downlink Spectral Efficiency with the Phaseaware MMSE Estimator . . . . . . . . . . . . . . 95

6.2 Non-Coherent Downlink Spectral Efficiency with the LMMSE and LS Estimator . . . . . . . . . . . . . . . . . . . . 96

$7 \quad$ Numerical Results . . . . . . . . . . . . . . . . . . . . . . . . . . . 97

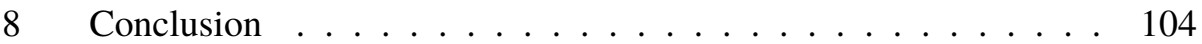

9 Appendix ............................. 105

9.1 Derivation of the LMMSE Estimator . . . . . . . . 105

9.2 Derivation of the LS Estimator . . . . . . . . . . 105

9.3 Proof of UL and DL SE with MMSE estimator . . . . . . 106

9.4 Proof of UL and DL SE with LMMSE Estimator . . . . . 110

9.5 Proof of UL and DL SE with the LS Estimator . . . . . . 112

9.6 Proof of DL SE with Non-coherent Transmission . . . . . 114

References . . . . . . . . . . . . . . . . . 115 



\section{Chapter 1}

\section{Introduction}

\subsection{Background and Motivation}

The initial understanding of radio wave propagation and the concept of wireless communication date back to the 19th century. In 1895, the entrepreneur Guglielmo Marconi successfully transmitted and received a coded message at a distance of 3.2 kilometers near his home in Bologna, Italy. Since then, the number of voice and data connections has doubled every 30 months [1]. Today, wireless connectivity has fundamentally changed the way we communicate and it is an integral part of our lives.

In its most elementary form, wireless communication is performed between a single transmitting antenna at the source and a single receiving antenna at the destination. This single-input single-output (SISO) wireless technology has been in use since the invention of wireless communication. However, the wireless propagation environments may pose severe challenges (see Section 1.2.1 for a more detailed explanation) and SISO is not robust to these challenging propagation conditions. To combat the channel impediments and receive a strong signal with SISO, a straightforward approach may be increasing the transmit power. However, this technique has two main drawbacks. First, the demanded mobile data volumes unprecedentedly inflate and it is not feasible to increase the transmit power boundlessly. Second, the number of connected devices unceasingly increases. Their signals would interfere with each other and the network may be easily pushed to an interference-limited regime. Instead of increasing the transmit power, an alternative approach is to increase the number of antennas at the transmitter or the receiver, or at both ends. By doing so, more power can be collected from the electromagnetic waves.

Remarkably in the 1920s, radio engineers H. H. Beverage and H. O. Peterson noticed that radio broadcast signals at two stations located about a half-mile apart had very different received signal strengths due to different levels of fading. To 
overcome this issue, they developed a multiple antenna system with two receiver antenna for each radio station. Further, their experimental observations indicated that these antennas needed to be separated by at least a wavelength for reliable operation [2]. Nevertheless, the initial deployment of multiple-input multiple-output (MIMO) systems started in the 1990s as the demand for more reliable wireless links and higher data rates grew.

MIMO technology has several prominent advantages over SISO in terms of array, diversity and multiplexing gains. Array gain means a power gain of the transmitted signal achieved using by multiple antennas at the transmitter and/or receiver compared with that of SISO systems. The usage of multiple antennas at transmitter enables directional beamforming that can be used to point the signal towards the user. Likewise, using multiple antennas at the receiver side provides a power gain since the receiver can collect more power from the transmitted signal. In addition, these antennas can provide a spatial diversity gain since they receive different copies of the same signal, which are unlikely to all be nearly zero simultaneously. The diversity can dramatically improve the performance when communicating under challenging channel conditions and provide more reliable wireless links. Moreover, having multiple antennas at the both ends provides an additional spatial dimension for communication and yields a multiplexing gain. The transmitter can separate the received signals in the spatial domain such that multiple parallel data streams can be supported on the same time and frequency resource. Spatial multiplexing of several data streams concurrently leads to an increase in the rate that is proportional to the number of data streams under ideal signal conditions.

As mentioned above, we observe an unceasingly increasing data traffic. This trend is expected to continue in the near future [3]. Massive MIMO technology is one of the key enablers of future cellular networks, by virtue of beamforming and spatial multiplexing. A massive MIMO base station is equipped with a massive number (e.g., a hundred) of individually steerable antennas, which can be effectively used to serve tens of user equipments simultaneously on the same time-frequency resource. It is relatively more convenient to deploy multiple antennas at the base station than at the users since the user equipments are generally compact, commercial and powered by small batteries and therefore less reliable products. Massive MIMO can provide higher data rates without the need for more bandwidth or dense deployment of base stations. It is an efficient way to achieve high spectral efficiency $(\mathrm{bit} / \mathrm{s} / \mathrm{Hz})$ per cell and per user. A massive MIMO base station can serve more users compared to a conventional MIMO base station and this results in a higher spectral efficiency per cell. Additionally, increased array gain and beamforming directivity reduce the inter-user interference that brings higher spectral efficiency per user.

After the seminal paper [4] providing the initial framework of massive MIMO with infinitely many antennas, the achievable spectral efficiencies of massive MIMO 
systems with practical number of antennas have been rigorously characterized and optimized by either spatially uncorrelated [5] or correlated Rayleigh fading [6, 7]. Further, massive MIMO communication with free-space line-of-sight (LoS) propagation, meaning that the environment is scatterer free and there is a direct path between the transmitter and the receiver, is treated in [8]. In massive MIMO research, i.i.d. Rayleigh fading or less commonly free-space LoS channel models are quite popular since they simplify the analysis. Massive MIMO technology is able to support both rich scattering and LoS scenarios. However, practical channels can consist of a combination of an LoS path (often called a specular path) and a correlated small-scale fading component caused by a finite number of scattering clusters that can be modeled by spatially correlated Rician fading (see Section 1.2.5 for a more detailed explanation). These channel models are discussed in detail in the later sections. The performance of massive MIMO with Rician fading channels are much less analyzed than the others. Paper A considers a multi-cell scenario with spatially correlated Rician fading channels and derives closed-form achievable spectral efficiency expressions for different signal processing techniques.

A radically different way of deploying a massive number of antennas is spreading them over a large geographical area $[9,10]$. This concept is called cell-free massive MIMO and has attracted lots of interest, see for example [11-15]. The idea of distributed antennas has been around for many years and various other names have been given to it in the past, such as network MIMO $[16,17]$ or cooperative multipoint (CoMP) $[18,19]$. Different from the network MIMO or CoMP, larger number of antennas are employed in cell-free massive MIMO and the benefits of massive MIMO are exploited. In the canonical form of cell-free system, the access points cooperate via a fronthaul network to spatially multiplex the users on the same time-frequency resource using network MIMO methods that only require locally obtained channel state information $[14,20]$. The main advantages of distributing the antennas are spatially diversity, increased coverage and possibility of providing uniform service.

Cell-free massive MIMO is a densely deployed system. Hence, the probability of having an LoS path between some access points and the users is quite high. Despite that, in early works, Rayleigh fading is assumed $[12,13]$. In Paper B, we consider a practical scenario where the channels between the access points and the users are modeled with Rician fading. Moreover, taking into account mobility of the users and hardware impairments, the LoS phase is modeled as a uniformly distributed random variable.

\subsection{Channel Models for Massive MIMO}

In this section, we discuss some common channel models for massive MIMO in further detail. In particular, we focus on the effects of spatial correlation and of the 
LoS path.

\subsubsection{Channel Fading}

In wireless communications, electromagnetic waves are designed to carry information from a source to a destination. The electromagnetic theory of light was formulated by James Clerk Maxwell in 1864. In his pioneering work, he predicted the existence of radio waves. Later on, Heinrich Rudolf Hertz proved the existence of these radio waves by conducting a series of experiments.

One of the main challenges in wireless communications is that the emitted waves often do not reach the receiving antenna directly due to the obstacles in the propagation environment. The received wave is a superposition of waves coming from many directions due to reflection, scattering, and diffraction caused by buildings, trees, cars, and other objects. Reflection occurs when the wave bounces off a smooth object that is exceedingly large compared to the wavelength. Then, each incoming wave is mapped onto a single beam. Scattering happens when the wave propagates through a medium that contains objects whose dimensions are smaller or comparable to the wavelength. Thus, each incoming wave is mapped onto many scattered ones. The radio waves can also be bent around to shape edges, such as rooftops, and this phenomenon is referred to as diffraction [21].

Ideally, the electromagnetic wave propagation can be exactly modeled by solving Maxwell's equations. In a fixed environment, this requires the calculation of radar cross sections of all surrounding objects. In a mobile environment, the obstacles change with time and their effects should also be taken into account. In general, these calculations are not computationally feasible and many required parameters are rarely available. Therefore, in wireless communications, Maxwell's equations are not used to model the propagation of transmitted signals. Instead, the wave is approximated as a ray-of-light and ray-tracing methods are employed. Based on this approximation, the ray propagation in different environments for various frequency bands are constructed by computer simulations. Some ray tracing methods attempt to reproduce the propagation mechanisms (reflection, scattering, and diffraction) in an actual physical location, for example see [22,23]. Other techniques create virtual scenarios for performance evaluation [24]. In realistic and complicated environments, the ray-based approaches often fail to model the propagation accurately or they can be computationally very demanding. In those cases, the empirical measurements and models can be utilized.

When a wave reaches the receiver by two or more paths, this phenomenon is called the multipath effect. The deterministic ray-tracing methods are useful when the number of paths is small. In general, the time-varying and complex nature of the radio channels prohibit to obtain an accurate model using deterministic methods. In 
practice, the number of paths is large and statistical models are utilized to characterize the constructive and destructive addition of different multipath components. The time-varying nature of the propagation channels stems from the movement of the transmitter and the receiver or the objects in the environment that changes the characteristics of the multipaths. Therefore, amplitudes and time delays of the transmitted waves unpredictably fluctuate over time.

The variations in the received power that is described above is called fading. The propagation effects that cause fading are classified as large-scale and small-scale effects. Path loss due to dissipation of the radiated power over the distance and shadow fading caused by obstacles that absorb power are referred to as large-scale fading effects. The fluctuations due to multipaths create the small-scale fading effects. These variations are referred to as small-scale fading because they occur when the user moves over short distances, at the order of the signal wavelength, whereas large-scale fading effects occur when the user moves over larger distances.

A distinct multipath component of the channel can be in the form of a single scatterer or a cluster of scatterers. In a multipath channel, when a single pulse is emitted from the transmitter, it is reflected by these scatterers. Due to this dispersive nature of the wireless channels, a pulse train is received at the destination with different time delays. The time delay between the first received and the last received pulses is referred to as the time delay spread of the channel. When the delay spread is large relative to the inverse of the signal bandwidth, the signal is scattered from different multipath components and interfere with subsequently transmitted pulses. This effect is called intersymbol interference and may lead a substantial signal distortion. These channels are referred to as wideband channels. If the delay spread is smaller than the inverse of the signal bandwidth then the received signal is less distorted and these channels are called narrowband channels.

A classical solution to the intersymbol interference problem is to divide the bandwidth into many subcarriers each having a sufficiently narrow bandwidth. The frequency interval $B_{c}$ over which the channel is approximately constant is referred to as the coherence bandwidth. Therefore, all frequency components of the signal experience the same magnitude of fading. Similarly, the coherence time $T_{c}$ describes the time interval in which the channel response is almost constant. Each resource block that consists of a number of subcarriers and time samples where the channel response can be approximated as constant and flat fading is called a coherence block. A coherent block contains $\tau_{c}=B_{c} T_{c}$ complex-valued samples. Throughout this thesis, we assume that the channel realizations are independent between any pair of blocks which is known as the block fading assumption. 


\subsubsection{Line-of-Sight Channels}

In this part, we define and explain features of an LoS channel. For the sake of argument, assume a propagation environment that is free from any reflectors or scatterers. The emitted waves travel in a direct path between the user and the base station. The base station is equipped with $M$ antennas and the user has a single antenna. The antennas can be arranged according to different array geometries. The basic one is the uniform linear array (ULA) in which the antennas are evenly spaced on a straight line. The antenna spacing between antennas (in wavelengths) is denoted by $d_{H}$. Then, the spacing between two adjacent antennas is $d_{H} \lambda$ where $\lambda$ is being the wavelength at the carrier frequency.

Suppose the user at a fixed location transmits the signal

$$
s(t)=\operatorname{Re}\left\{u(t) e^{j 2 \pi f_{c} t}\right\}
$$

where $u(t)$ is the complex baseband signal and $f_{c}$ is the carrier frequency. Then, ignoring the noise, the received signal at the $m$ th antenna of the base station ULA can be written as a convolution of the transmitted bandpass signal $s(t)$ and channel impulse response $h_{m}(t)$ as [25, Section 7.2 ]

$$
\begin{aligned}
r_{m}(t)=\left(h_{m} * s\right)(t) & =\operatorname{Re}\left\{\sqrt{\beta^{m}} u\left(t-T_{m}\right) e^{j 2 \pi f_{c}\left(t-T_{m}\right)}\right\} \\
& =\operatorname{Re}\left\{\left(\int_{-\infty}^{\infty} h_{m}(T) u(t-T) d T\right) e^{j 2 \pi f_{c} t}\right\}
\end{aligned}
$$

where " $*$ " denotes the convolution operator, $T_{m}$ is the time delay associated with the direct path between the user and the $m$ th antenna. The coefficient $\beta^{m}$ describes the large-scale fading effects that account for dissipation of the transmit power over the distance between the base station antenna $m$ and the user. Hence, the channel response of $m$ th path is

$$
h_{m}(T)=\sqrt{\beta^{m}} e^{-j 2 \pi f_{c} T_{m}} \delta\left(t-T_{m}\right)=\sqrt{\beta^{m}} e^{-j 2 \pi f_{c} \frac{d_{m}}{c}} \delta\left(t-T_{m}\right)
$$

where $c$ is the speed of light and $d_{m}$ is the distance between the antenna $m$ and the user. If $d_{m}$ is larger than the Fraunhofer distance $\frac{2 D^{2}}{\lambda}$, where $D$ is the largest dimension of the antenna array, that defines the limit between the geometric near and far field regions, it is reasonable to assume that the incoming wave has a planar wavefront. Since the propagation distance is much larger than the size of ULA at BS, we can write

$$
d_{m} \approx d-(m-1) d_{H} \lambda \sin (\varphi)
$$

where $d$ is the largest distance from the user to the base station and $\varphi$ is the angle of incidence onto the antenna array as depicted in Figure 1. Then, in a coherence 

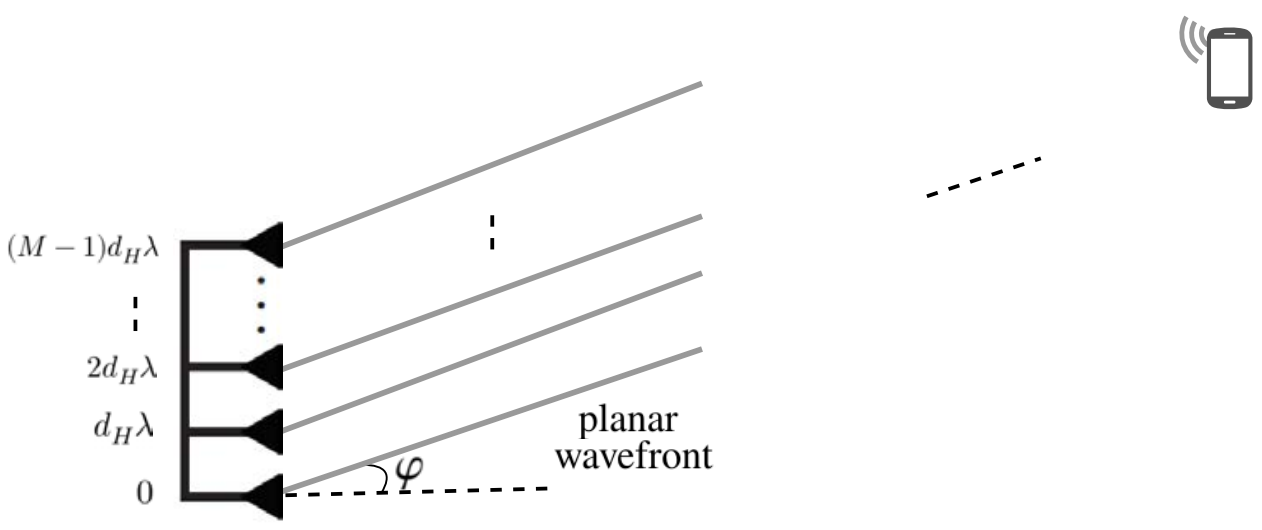

Figure 1: LoS propagation between a transmitting single antenna and a base station equipped with a ULA.

block, the channel response at the $m$ th antenna is

$$
h_{m}=\sqrt{\beta^{m}} e^{\frac{-j 2 \pi d}{\lambda}} e^{j 2 \pi d_{H}(m-1) \sin (\varphi)} .
$$

The large-scale fading terms $\sqrt{\beta^{m}} e^{\frac{-j 2 \pi d}{\lambda}}=\sqrt{\beta}$, for all $m$, neglecting $e^{\frac{-j 2 \pi d}{\lambda}}$ since it takes an arbitrary value on the unit circle that is the same for all antennas and $d$ is large compared to the size of ULA. Stacking the channel coefficients into a vector gives

$$
\mathbf{h}=\sqrt{\beta}\left[1 e^{j 2 \pi d_{H} \sin (\varphi)} \ldots e^{j 2 \pi d_{H}(M-1) \sin (\varphi)}\right]^{T} .
$$

A massive MIMO base station is equipped with a massive number of antennas (e.g., a hundred), and it is able to serve multiple users thanks to its high resolution in the angular domain. To illustrate this property, suppose that the base station ULA is horizontally deployed. It serves two users that are located at the same elevation angle but the different azimuth angles $\varphi_{1}$ and $\varphi_{2}$ respectively. The magnitude of the inner product of their channel vectors can be computed as

$$
\left|\mathbf{h}_{1}^{H}\left(\varphi_{1}\right) \mathbf{h}_{2}\left(\varphi_{2}\right)\right|=\sqrt{\beta_{1} \beta_{2}} g\left(\varphi_{1}, \varphi_{2}\right)
$$

where

$$
g\left(\varphi_{1}, \varphi_{2}\right)=\left|\sum_{m=1}^{M} e^{j 2 \pi d_{H}(m-1)\left(\sin \left(\varphi_{1}\right)-\sin \left(\varphi_{2}\right)\right)}\right|
$$

Using the identity $\sum_{n=0}^{N-1} q^{n}=\frac{1-q^{N}}{1-q}$ for $q \neq 1$, we obtain $g\left(\varphi_{1}, \varphi_{2}\right)=\left|\frac{1-e^{j 2 \pi M d_{H}\left(\sin \left(\varphi_{1}\right)-\sin \left(\varphi_{2}\right)\right)}}{1-e^{j 2 \pi d_{H}\left(\sin \left(\varphi_{1}\right)-\sin \left(\varphi_{2}\right)\right)}}\right|=\left|\frac{\sin \left(\pi M d_{H}\left(\sin \left(\varphi_{1}\right)-\sin \left(\varphi_{2}\right)\right)\right)}{\sin \left(\pi d_{H}\left(\sin \left(\varphi_{1}\right)-\sin \left(\varphi_{2}\right)\right)\right)}\right|$. 


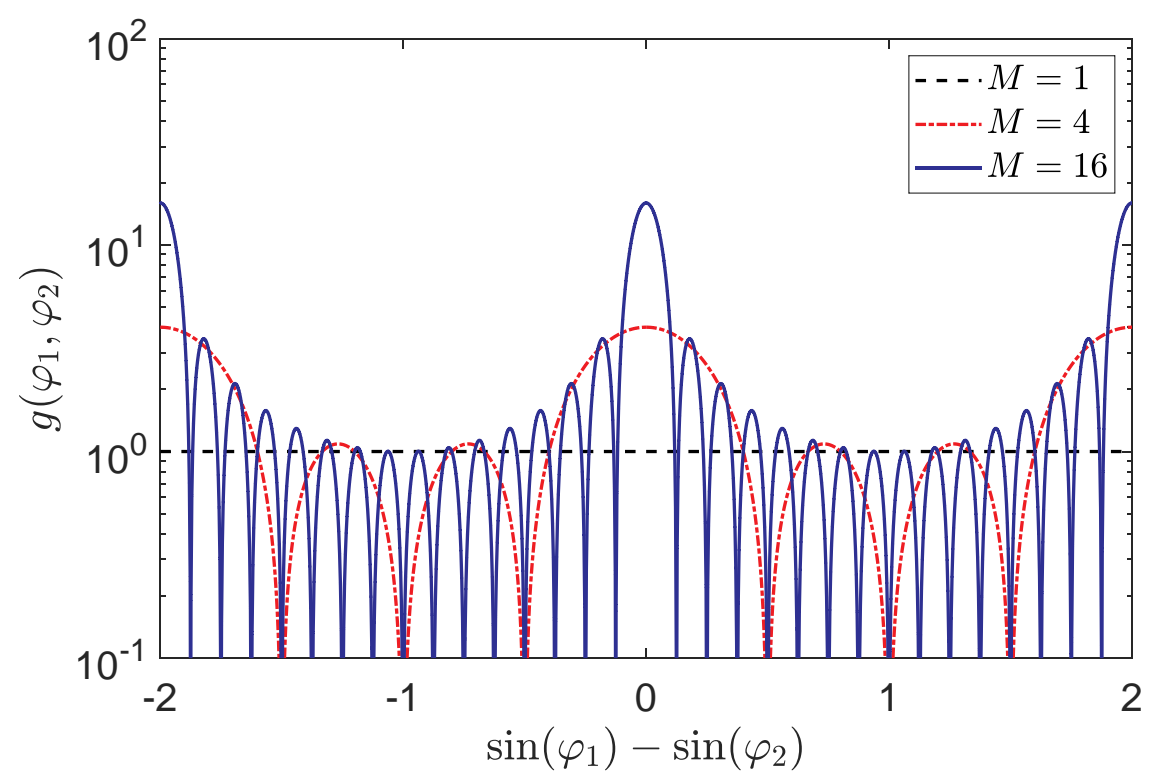

Figure 2: The function $g\left(\varphi_{1}, \varphi_{2}\right)$ versus difference of the user angles for different number of ULA antennas where $d_{H}=0.5$.

From Figure 2, one can observe that the function $g\left(\varphi_{1}, \varphi_{2}\right)$ has the following properties:

- It peaks at $\sin \left(\varphi_{1}\right)=\sin \left(\varphi_{2}\right): g\left(\varphi_{1}, \varphi_{2}\right)=M$.

- $g\left(\varphi_{1}, \varphi_{2}\right)=0$ at $\sin \left(\varphi_{1}\right)-\sin \left(\varphi_{2}\right)=\frac{m}{M d_{H}}$, for $m=1, \ldots, M-1$.

- It is periodic with period $\frac{1}{d_{H}}$.

The values of $\sin \left(\varphi_{1}\right)-\sin \left(\varphi_{2}\right)$ are confined within an interval $[-2,2]$. The main lobes of $g\left(\varphi_{1}, \varphi_{2}\right)$ have a width of $\frac{2}{M d_{H}}$ and they are centered around the integer multiples of $\frac{1}{d_{H}}$. Therefore, $\varphi_{1}$ and $\varphi_{2}$ are not distinguishable by the ULA whenever

$$
\left|\sin \left(\varphi_{1}\right)-\sin \left(\varphi_{2}\right)\right| \ll \frac{1}{M d_{H}} .
$$

for $d_{H} \leq 0.5$. The parameter $\frac{1}{M d_{H}}$ can be seen as a measure of resolvability in the angular domain. Note that it is inversely proportional to the width of the array, which means that massive MIMO has a good angular resolvability. Another important point is that keeping the length of ULA (proportional to $M d_{H}$ ) fixed and increasing the number of packed antennas does not increase the resolvability since it forces $d_{H} \rightarrow 0$. 


\subsubsection{Spatially Uncorrelated Rayleigh Fading}

In the last subsection, we discussed an LoS channel model in a static free space environment which means that there was no scatterer or reflector in the propagation environment. Besides, both the user and the base station were static during transmission. These are rather extreme assumptions that rarely occur on earth in practice. Nevertheless, it may be a good model in space.

In this subsection, we consider another extreme case: the uncorrelated Rayleigh fading model. Assume that we have the same base station and the user from the previous subsection. However, this time, we assume rich scattering conditions where the propagation environment contains many scatterers compared to the number of the base station antennas. In addition, the user and/or the scatterers may change their locations. Then, the transmitted signal from the user reaches the ULA through many different paths.

Suppose, for ease of notation, the mobile user sends an unmodulated carrier signal $s(t)=\operatorname{Re}\left\{e^{j 2 \pi f_{c} t}\right\}=\cos \left(j 2 \pi f_{c} t\right)$ to the base station equipped with the $M$ antenna ULA. Note that $s(t)$ is narrowband for any multipath time delay spread. This signal propagates through a rich scattering environment with $N$ multipath components. Then, neglecting the receiver noise, the received signal at the antenna $m$ can be written as $r_{m}(t)=\left(h_{m} * s\right)(t)=\operatorname{Re}\left\{h_{m}(t) e^{j 2 \pi f_{c} t}\right\}$ where $h_{m}(t)$ models the effects of the propagation environment. It is the superposition of responses from all paths and can be described as [26]

$$
h_{m}(t)=\sum_{n=1}^{N} \alpha_{m, n} e^{-j \phi_{m, n}(t)} \delta\left(t-T_{m, n}\right)
$$

where $\alpha_{m, n} \in \mathbb{R}$ is the gain, $T_{m, n}$ is the time delay and $\phi_{m, n}(t)$ accounts for the phase rotation associated with the $n$th path. To characterize the varying nature of the propagation environment, the path gain $\alpha_{m, n}$ and phase rotation $\phi_{m, n}(t)$ can be modeled as independent and identically distributed (i.i.d.) random variables. If $N$ is large enough the central limit theorem implies that $h_{m}(t)$ is a circularly symmetric complex Gaussian variable and therefore the fading envelope $\left|h_{m}(t)\right|$ is Rayleigh distributed. Recall that a random variable $Z$ is Rayleigh distributed (i.e., Rayleigh $(\sigma)$ ) if $Z=\sqrt{X^{2}+Y^{2}}$ where $X \sim \mathcal{N}\left(0, \sigma^{2}\right)$ and $Y \sim \mathcal{N}\left(0, \sigma^{2}\right)$ are independent. The Rayleigh distribution has probability density function [27]

$$
p_{Z}(z)=\frac{z}{\sigma^{2}} e^{-\frac{z^{2}}{2 \sigma^{2}}}, \quad z \geq 0 .
$$

The early studies $[28,29]$ state that the Rayleigh distribution approximation is quite accurate when $N \geq 6$.

The path gain $\alpha_{m, n}$ depends on the large scale effects such as the dissipation and absorption of the signal power over large distances, namely path loss and shadowing. 
On the other hand, the phase shift term $\phi_{m, n}(t)$ can change quickly, on the order of a signal wavelength, due to constructive and destructive additions of different multipath components. Thus, typically, $\alpha_{m, n}$ varies relatively slower than $\phi_{m, n}(t)$ and we assume that $\alpha_{m, n}$ is constant for many coherence blocks whereas $\phi_{m, n}(t)$ changes in every coherence block.

There are several effects that can cause the phase rotation. For example, even in free space when the user moves relative to the base station, the frequency of the propagating waves change. More specifically, if the user (source of the waves) moves towards the base station, each successive wave front is emitted from a position closer to the base station. Therefore, the wavefront reaches the base station in less time. The time between the arrival of each successive wave crest is reduced i.e., the frequency is increased. Conversely, if the user moves away from the base station, each wave is emitted from a position farther from the base station than the previous wave, so the arrival time between successive waves is increased, reducing the frequency. This phenomena is called Doppler shift and here denoted with $f_{D}$. To quantify $f_{D}$ suppose in a time interval $\Delta t$, the change in the distance between the user and the base station is $\Delta d=v \Delta t \cos (\varphi)$ where $v$ is the velocity of the user (assuming that it follows a linear trajectory) and $\varphi$ is the angle of arrival to the base station relative to the direction of motion. The corresponding phase change due to this path length difference is $\Delta \phi_{D}=\frac{\Delta d}{\lambda}=\frac{2 \pi v \Delta t \cos (\varphi)}{\lambda}$. Then, the Doppler frequency is obtained from the relation $f_{D}=\frac{1}{2 \pi} \frac{\Delta \phi_{D}}{\Delta t}=\frac{v \cos (\varphi)}{\lambda}$. Throughout this section, we assume that $v$ is changing slowly enough (i.e., small or zero acceleration) such that it is constant over the time interval of interest. If it is not the case then $f_{D}(t)$ is a function of time and the corresponding Doppler phase shift becomes $\phi_{D}(t)=\int_{0}^{t} 2 \pi f_{D}(t) d t$.

In a multipath environment, each scattering path may introduce a different amount of Doppler frequency shift, $f_{D_{m, n}}=\frac{v \cos \left(\varphi_{m, n}\right)}{\lambda}$. The difference between the Doppler shifts of two paths is called the Doppler spread. The maximum of these differences is referred to as the maximum Doppler spread of the channel and here denoted by $B_{D}$. It is a measure of the channel frequency dispersion and inversely proportional to the channel coherence time $T_{c} \approx \frac{1}{B_{D}}$.

Another effect that can cause the phase rotation is the multipath time delays. To illustrate this behavior, suppose a carrier frequency $f_{c}=3 \mathrm{GHz}$ and a multipath time delay associated to the path $n$ and the antenna $m, T_{m, n}=60 \mathrm{~ns}$ (a typical value for an indoor environment). The resulting phase rotation is $2 \pi f_{c} T_{m, n}=0^{\circ}$. However, when this delay slightly changes, for example to $T_{m, n}=60.5 \mathrm{~ns}$, the phase variation becomes $180^{\circ}$. Note that outdoor systems generally have much larger multipath delays than $60 \mathrm{~ns}$, typically at order of milliseconds. It shows that since $f_{c}$ is generally large, a small change in $T_{m, n}$ can cause a rotation through $360^{\circ}$. Therefore, it is reasonable to assume that $2 \pi f_{c} T_{m, n}$ is uniformly distributed on $[-\pi, \pi]$. 
In addition to Doppler phase shifts and the multipath delays, the phase rotation term $\phi_{m, n}$ can have an initial phase offset $\phi_{0}$ that stems from the oscillators at the transmitter and the receiver [30]. Therefore, we can model the phase rotation term as

$$
\phi_{m, n}(t)=2 \pi f_{c} T_{m, n}+2 \pi f_{D_{m, n}} t+\phi_{0} .
$$

The uncorrelated Rayleigh fading channels appear under extremely strict physical requirements. To illustrate this, consider a uniform scattering environment where many scatterers are densely packed with equally spaced angles on a sphere around the base station ULA. The uniform scattering environment model was first proposed by Clarke [31] and then popularized by Jakes [32]. In this environment, the received power from all scatterers are assumed to be equal which implies $\mathbb{E}\left\{\alpha_{m, n}^{2}\right\}=\frac{P_{r}}{N}$ where $P_{r}$ is the total received power. This model implies that the channel vector $\mathbf{h}$ has no dominant spatial directivity since the signals are equally likely to arrive to the base station from any direction. Thus, each antenna receives an independent fading from other antennas i.e., $\mathbb{E}\left\{h_{m}^{*} h_{s}\right\}=0$ for $s \neq m$. It leads the uncorrelated Rayleigh fading model

$$
\mathbf{h} \sim \mathcal{N}_{\mathbb{C}}\left(\mathbf{0}_{M}, \beta \mathbf{I}_{M}\right)
$$

where $\beta$ accounts for the large-scale fading effects and the Gaussian distribution models the small-scale fading. With this model, the channel gain $\|\mathbf{h}\|^{2}$ is chi square distributed and is independent of the channel direction $\frac{\mathbf{h}}{\|\mathbf{h}\|}$ that is uniformly distributed over the unit sphere in $\mathbb{C}^{M}[6]$.

\subsubsection{Spatially Correlated Rayleigh Fading}

In the previous subsection, we discussed the i.i.d. Rayleigh fading model and assumed a uniform scattering environment in which the base station ULA was surrounded by a large number of scatterers. However, in practice, the base station is typically at an elevated position compared to the users at the ground. Thus, the base station rarely has scatterers in its close vicinity. In contrast, the scatterers are generally localized around the users and practical massive MIMO channels are spatially correlated as seen from measurement campaigns [33-35].

Suppose, as in the previous subsection, the ULA receives scattered signals from $N$ different multipath components but this time the scatterers are concentrated around the user. The channel between the base station antenna $m$ and the user is the superposition of responses from all paths and can be described as

$$
h_{m}(t)=\sum_{n=1}^{N} \alpha_{m, n} e^{-j \phi_{m, n}(t)} \delta\left(t-T_{m, n}\right)
$$

where $\alpha_{m, n} \in \mathbb{R}$ is the gain and $\phi_{m, n}(t)$ accounts for the phase rotation due to multipath delays, Doppler effect and initial phase offset associated with the $n$th path. 
We can rewrite (15) as

$$
\begin{aligned}
h_{m}(t) & =\sum_{n=1}^{N} \alpha_{m, n} e^{-j\left(2 \pi f_{c} T_{m, n}+2 \pi f_{D_{m, n}} t+\phi_{0}\right)} \delta\left(t-T_{m, n}\right) \\
& \stackrel{(\mathrm{a})}{=} \sum_{n=1}^{N} \alpha_{m, n} e^{-j\left(2 \pi f_{D_{m, n}} t+\phi_{0}\right)} e^{-j 2 \pi f_{c} \frac{d_{m, n}}{c}} \delta\left(t-T_{m, n}\right) \\
& \stackrel{(\mathrm{b})}{=} \sum_{n=1}^{N} \alpha_{m, n} e^{-j\left(2 \pi f_{D_{m, n}} t+\phi_{0}+2 \pi f_{c} \frac{d_{n}}{c}\right)} e^{j 2 \pi f_{c} \frac{(m-1) d_{H} \lambda \sin \left(\bar{\varphi}_{n}\right)}{c}} \delta\left(t-T_{m, n}\right) \\
& \stackrel{(\mathrm{c})}{=} \sum_{n=1}^{N} g_{n} e^{j 2 \pi(m-1) d_{H} \sin \left(\bar{\varphi}_{n}\right)} \delta\left(t-T_{m, n}\right)
\end{aligned}
$$

where $(a)$ follows from $T_{m, n}=\frac{d_{m, n}}{c}$ where $d_{m, n}$ is the distance between the antenna $m$ and the user through $n$th path. In $(b)$, using the far-field approximation, we substitute $d_{m, n} \approx d_{n}-(m-1) d_{H} \lambda \sin \left(\bar{\varphi}_{n}\right)$ where $d_{n}$ is the largest distance from the user to the ULA and $\bar{\varphi}_{n}$ is the angle of incidence onto the antenna array from the path $n$. In $(c)$, we note that the gain and phase rotations for each antenna is approximately the same since the size of the antenna array is much smaller than the propagation distance. Then, $\alpha_{m, n} \approx \alpha_{n}$ and we define $g_{n}=\alpha_{n} e^{-j\left(2 \pi f_{D_{m, n}} t+\phi_{0}+2 \pi f_{c} \frac{d_{n}}{c}\right)}$ for all $m \in 1, \ldots, M$.

Under the block flat fading assumption, we can drop the time index $t$ and write the channel response $\mathbf{h} \in \mathbb{C}^{M}$ as

$$
\mathbf{h}=\sum_{n=1}^{N} g_{n}\left[1, \ldots, e^{j 2 \pi(m-1) d_{H} \sin \left(\bar{\varphi}_{n}\right)}, \ldots, e^{j 2 \pi(M-1) d_{H} \sin \left(\bar{\varphi}_{n}\right)}\right]^{T}
$$

where $g_{n}$ represents both the gain and the phase rotation associated with the path $n$. Based on the same reasoning in the previous sections, we can model $g_{n}$ and the angle of arrivals $\bar{\varphi}_{n}$ as i.i.d. random variables. Suppose $\bar{\varphi}_{n}$ has probability density function $f(\bar{\varphi})$ and $g_{n}$ has zero-mean and variance $\mathbb{E}\left\{\left|g_{n}\right|^{2}\right\}$. The variance accounts for the average gain of the path $n$ and the total average gain of the all multipath components is denoted by $\beta=\sum_{n=1}^{N} \mathbb{E}\left\{\left|g_{n}\right|^{2}\right\}$. As the number of multipaths $N \rightarrow \infty$, the multidimensional central theorem implies that

$$
\mathbf{h} \rightarrow \mathcal{N}_{\mathbb{C}}\left(\mathbf{0}_{M}, \mathbf{R}\right)
$$

where the convergence is in distribution and the $(l, m)$ th element of correlation matrix $\mathbf{R}$ is

$$
\begin{aligned}
{[\mathbf{R}]_{l, m} } & =\sum_{n=1}^{N} \mathbb{E}\left\{\left|g_{n}\right|^{2}\right\} \mathbb{E}\left\{e^{j 2 \pi(l-1) d_{H} \sin \left(\bar{\varphi}_{n}\right)} e^{-j 2 \pi(m-1) d_{H} \sin \left(\bar{\varphi}_{n}\right)}\right\} \\
& =\beta \int e^{j 2 \pi(l-m) d_{H} \sin (\bar{\varphi})} f(\bar{\varphi}) d \bar{\varphi}
\end{aligned}
$$




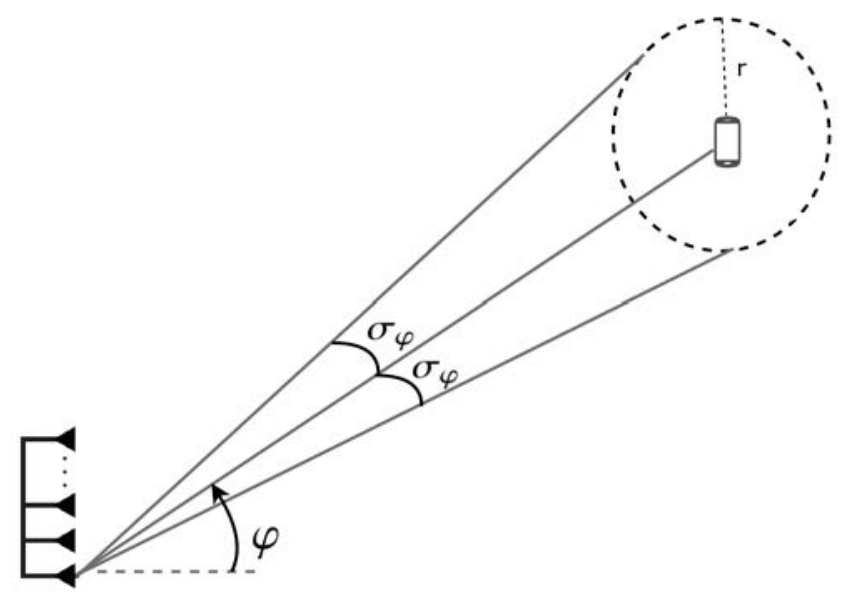

Figure 3: An illustration of the one-ring model.

The correlation matrix $\mathbf{R}$ can be computed for any angle distribution $f(\bar{\varphi})$. From (19), we can observe that the integral only depends on the difference $l-m$, not on the individual values of $l$ and $m$. It implies that $\mathbf{R}$ is a Toeplitz matrix.

The random arrival angle can be modeled as $\bar{\varphi}=\varphi+\delta$ where $\varphi$ is the deterministic nominal arrival angle and $\delta$ is the random deviation from this angle with variance $\sigma_{\varphi}^{2}$. The parameter $\sigma_{\varphi}$ is called angular standard deviation (ASD) and it is a measure of how large the deviations from the nominal angle are. In literature, there are different assumptions on the distribution of $\delta$. Mainly three probability distributions are studied: the uniformly distributed $\delta \sim \mathcal{U}\left[-\sigma_{\varphi}, \sigma_{\varphi}\right]$ [36-39], the Laplace distributed $\delta \sim \operatorname{Lap}\left(0, \frac{\sigma_{\varphi}}{\sqrt{2}}\right)[40,41]$ and the Gaussian distributed $\delta \sim \mathcal{N}\left(0, \sigma_{\varphi}^{2}\right)[39,42,43]$.

The uniformly distributed $\delta$ is also called the one-ring model. It is assumed that the scatterers around the user are uniformly located on a ring as shown in Figure 3. If the user is $d$ meters away from the base station and the ring has a radius $r$ then $\sigma_{\varphi} \approx \arctan \left(\frac{r}{d}\right)$. Then, the distribution of $\delta$ is

$$
f(\delta)= \begin{cases}\frac{1}{2 \sigma_{\varphi}}, & -\sigma_{\varphi} \leq \delta \leq \sigma_{\varphi} \\ 0, & \text { elsewhere }\end{cases}
$$

Inserting (20) into the integral (19) gives

$$
[\mathbf{R}]_{l, m}=\beta \int_{-\sigma_{\varphi}}^{\sigma_{\varphi}} \frac{1}{2 \sigma_{\varphi}} e^{j 2 \pi(l-m) d_{H} \sin (\varphi+\delta)} d \delta .
$$

The closed-form expression for this integral utilizing Bessel functions is provided in [44]. It is worth noting that the one-ring model is different from the uniform 
scattering environment assumption in the uncorrelated Rayleigh fading case. In the one-ring model, the scatterers are in the vicinity of the user whereas the base station is surrounded by the scatterers in the latter.

Alternatively, with the Laplacian angular spread model $\delta \sim \operatorname{Lap}\left(0, \frac{\sigma_{\varphi}}{\sqrt{2}}\right)$, the probability density function $f(\delta)=\frac{1}{\sqrt{2} \sigma_{\varphi}} e^{-\frac{\sqrt{2}|\delta|}{\sigma_{\varphi}}}$ and the integral (19) becomes [41]

$$
[\mathbf{R}]_{l, m}=\beta \int_{-\pi}^{\pi} \frac{1}{\sqrt{2} \sigma_{\varphi}} e^{-\frac{\sqrt{2}|\delta|}{\sigma_{\varphi}}} e^{j 2 \pi(l-m) d_{H} \sin (\varphi+\delta)} d \delta
$$

and this integral can be numerically calculated. Note that $\mathbf{R}$ is a Toeplitz matrix, so that it is enough to compute the first column (or row) and then we know all the entries. In the case of Gaussian angular spread model, $\delta \sim \mathcal{N}\left(0, \sigma_{\varphi}^{2}\right)$, the integral (19) is

$$
[\mathbf{R}]_{l, m}=\beta \int e^{j 2 \pi(l-m) d_{H} \sin (\varphi+\delta)} \frac{1}{\sqrt{2 \pi} \sigma_{\varphi}} e^{-\frac{\delta^{2}}{2 \sigma_{\varphi}^{2}}} d \delta .
$$

When the ASD is small enough $\left(\sigma_{\varphi} \leq 15^{\circ}\right), \sin (\delta) \approx \delta$ and $\cos (\delta) \approx 1$ [45] and we can approximately calculate (23) to reduce computational complexity as

$$
\begin{aligned}
{[\mathbf{R}]_{l, m} } & \approx \beta \int e^{j 2 \pi(l-m) d_{H} \sin (\varphi)} e^{j 2 \pi(l-m) d_{H} \cos (\varphi) \delta} \frac{1}{\sqrt{2 \pi} \sigma_{\varphi}} e^{-\frac{\delta^{2}}{2 \sigma_{\varphi}^{2}}} d \delta \\
& =\beta e^{j 2 \pi(l-m) d_{H} \sin (\varphi)} e^{\frac{-\sigma_{\varphi}^{2}}{2}\left(2 \pi(l-m) d_{H} \cos (\varphi)\right)^{2}} \int \frac{1}{\sqrt{2 \pi} \sigma_{\varphi}} e^{-\frac{\left(\delta-j \sigma_{\varphi}^{2} 2 \pi(l-m) d_{H} \cos (\varphi)\right)^{2}}{2 \sigma_{\varphi}^{2}}} d \delta \\
& =\beta e^{j 2 \pi(l-m) d_{H} \sin (\varphi)} e^{\frac{-\sigma_{\varphi}^{2}}{2}\left(2 \pi(l-m) d_{H} \cos (\varphi)\right)^{2}}
\end{aligned}
$$

where the last equality follows from $\int \frac{1}{\sqrt{2 \pi} \sigma_{\varphi}} e^{-\frac{\left(\delta-j \sigma_{\varphi}^{2} 2 \pi(l-m) d_{H} \cos (\varphi)\right)^{2}}{2 \sigma_{\varphi}^{2}}} d \delta=1$. Note that in the extreme case of $\sigma_{\varphi}=0$, the signal comes from a fixed direction (with angle of arrival $\varphi$ ) and the correlation matrix has rank 1. Conversely, as $\sigma_{\varphi}$ grows off-diagonal elements of $\mathbf{R}$ go to zero and the rank increases.

The eigenstructure of $\mathbf{R}$ determines which spatial directions are statistically more likely to contain signal components than others. Suppose that $\mathbf{R}$ has the eigenvalue decomposition $\mathbf{R}=\mathbf{U} \boldsymbol{\Lambda} \mathbf{U}^{H}$ where $\boldsymbol{\Lambda} \in \mathbb{R}^{r \times r}$ is a diagonal matrix containing $r=\operatorname{rank}(\mathbf{R})$ non-zero eigenvalues of $\mathbf{R}$ and $\mathbf{U} \in \mathbb{C}^{M \times r}$ consists of the associated eigenvectors such that $\mathbf{U}^{H} \mathbf{U}=\mathbf{I}_{r}$. Then, using the Karhunen-Loéve representation [46], we can write the channel vector $\mathbf{h}$ as

$$
\mathbf{h}=\mathbf{R}^{1 / 2} \mathbf{w}=\mathbf{U} \boldsymbol{\Lambda}^{1 / 2} \mathbf{U}^{H} \mathbf{W} \sim \mathbf{U} \boldsymbol{\Lambda}^{1 / 2} \hat{\mathbf{w}}
$$




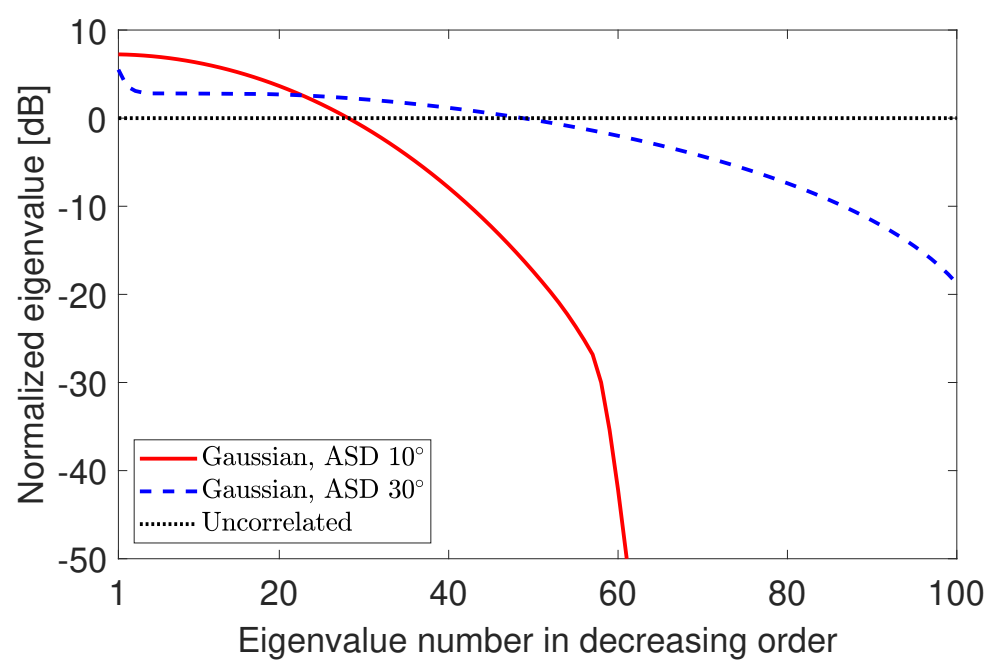

Figure 4: Normalized eigenvalues of the spatial correlation matrix $\mathbf{R}$ where $\operatorname{tr}(\mathbf{R})=$ $M$ with $M=100$, nominal angle $\varphi=30^{\circ}$ and standard deviations $\sigma_{\varphi}=\left[10^{\circ}, 30^{\circ}\right]$. The uncorrelated fading is added as a reference where $\mathbf{R}=\mathbf{I}_{M}$.

where $\mathbf{w} \sim \mathcal{N}_{\mathbb{C}}\left(\mathbf{0}_{M}, \mathbf{I}_{M}\right)$ and $\hat{\mathbf{w}} \sim \mathcal{N}_{\mathbb{C}}\left(\mathbf{0}_{r}, \mathbf{I}_{r}\right)$. In the last step, " $\sim$ " implies that the distributions of $\mathbf{h}$ and $\mathbf{U} \boldsymbol{\Lambda}^{1 / 2} \hat{\mathbf{w}}$ are identical. This representation is a convenient way to generate fading realizations of a spatially correlated channel.

According to the Szegò's theorem, the Toeplitz matrix $\mathbf{R}$ becomes asymptotically equivalent to a circulant matrix as $M \rightarrow \infty$ [47]. Then, its eigenvector matrix is equal to the unitary discrete Fourier transform (DFT) matrix $\mathbf{F} \in \mathbb{C}^{M \times M}$ with elements

$$
[\mathbf{F}]_{l, m}=\frac{1}{\sqrt{M}} e^{j \frac{2 \pi}{M}(l-1)(m-1)}
$$

for $l, m \in 1, \ldots, M$. In the regime of a large number of antennas, we can approximate $\mathbf{U}$ in (25) with $\overline{\mathbf{F}} \in \mathbb{C}^{M \times r}$ that is a submatrix formed by selection of $r$ columns from $\mathbf{F}$. Thus, $\mathbf{R} \approx \overline{\mathbf{F}} \boldsymbol{\Lambda} \overline{\mathbf{F}}^{H}$ and this can also be used to generate spatially correlated fading realizations instead of the Karhunen-Loéve representation. If the antennas in the ULA are critically spaced i.e., $d_{H}=0.5$ then $\overline{\mathbf{F}}^{H} \mathbf{h}$ is equivalent to the angular domain representation [25, Chapter 7.3.3].

High levels of spatial correlation occurs when $\sigma_{\varphi}$ is small. As shown in Figure 4 , higher spatial correlation creates larger eigenvalue variations. In the case of $\sigma_{\varphi}=10^{\circ}, \mathbf{R}$ has a low rank since almost $60 \%$ of the eigenvalues are negligibly small compared to others. It means that the propagation environment has some dominant spatial eigenvectors. As $\sigma_{\varphi}$ grows, the spatial directions spread and variation in the 
eigenvalues becomes smaller. In the uncorrelated fading case, $\mathbf{R}$ is full-rank and has no spatial directivity.

The local scattering channel models discussed above are mathematically convenient and easy to simulate while still capturing essential characteristics of the wireless channel between the base station equipped with large antenna arrays and single-antenna users. However, these models may be insufficient for large-scale system-level simulations with the goal of quantifying the real-world performance of MIMO systems. In particular, it is not realistic to assume a single local cluster of scatterers around the user. For this reason, the 3rd Generation Partnership Project (3GPP) has defined a 3D stochastic geometry-based channel model for MIMO systems [48], which explicitly accounts for multiple spatial clusters of scatterers and a mix of NLoS and LoS propagation paths. In Paper A, based on the 3GPP model in [48], we consider $N_{c}=6$ disjoint scattering clusters where each cluster is modeled by the (approximate) Gaussian local scattering model such that

$$
[\mathbf{R}]_{l, m}=\frac{\beta}{N_{c}} \sum_{n=1}^{N_{c}} e^{\jmath 2 \pi(l-m) d_{H} \sin \left(\varphi_{n}\right)} e^{-\frac{\sigma_{\varphi}^{2}}{2}\left(2 \pi(l-m) d_{H} \cos \left(\varphi_{n}\right)\right)^{2}},
$$

where $\varphi_{n} \sim \mathcal{U}\left[\varphi-40^{\circ}, \varphi+40^{\circ}\right]$ is the nominal AoA for the $n$ cluster. The multipath components of a cluster have Gaussian distributed AoAs, distributed around the nominal AoA with the $\operatorname{ASD} \sigma_{\varphi}=5^{\circ}$.

\subsubsection{Spatially Correlated Rician Fading}

The i.i.d. Rayleigh fading or pure LoS channel models are widely used in massive MIMO research since they simplify the analysis. However, practical channels can consist of a combination of an LoS path and a correlated small-scale fading component caused by a finite number of scattering clusters. In this subsection, we consider the spatially correlated Rician fading model that accounts for these effects.

Suppose that the base station is equipped with the ULA and the mobile user has a single antenna as before. In this case, the propagation channel between the base station and the user can be modeled as

$$
\mathbf{h}=\overline{\mathbf{h}} e^{j \theta}+\mathbf{g}
$$

where $\overline{\mathbf{h}} e^{j \theta} \in \mathbb{C}^{M}$ corresponds to the LoS component and $\mathbf{g} \sim \mathcal{N}_{\mathbb{C}}(\mathbf{0}, \mathbf{R})$ accounts for the non-line-of-sight (NLoS) (often referred to as diffuse) components. The macroscopic propagation effects of the LoS is described by the term $\overline{\mathbf{h}} \in \mathbb{R}^{M}$ and the phase rotation due to mobility of the user is denoted by $e^{j \theta}$ where $\theta \sim \mathcal{U}[-\pi, \pi]$. Note that different from the static LoS model discussion in Section 1.2.2, here the user moves during the transmission and the mobility of user introduces the phase rotation due to the effects such as time delay and Doppler phase shift. 
Each element of $\mathbf{h}$ is denoted by $h_{m}$ for $m \in\{1, \ldots, M\}$ and the channel envelope $\left|h_{m}\right|$ is Rice distributed. The channel model of $\mathbf{h}$ is named after Stephan O. Rice as Rician or Ricean fading. In the literature, there is no agreement on the spelling. Today, a Google scholar search gives 47.3k results for "Rician" and 14.3k for "Ricean". Therefore, we preferred to use "Rician" in this thesis.

A random variable $Z$ is Rice distributed (i.e., $Z \sim \operatorname{Rice}(|v|, \sigma))$ if $Z=$ $\sqrt{X^{2}+Y^{2}}$ where $X \sim \mathcal{N}\left(v \cos (\theta), \sigma^{2}\right)$ and $Y \sim \mathcal{N}\left(v \sin (\theta), \sigma^{2}\right)$ are independent [49]. For this parameters, the probability distribution is given by [49]

$$
p_{Z}(z)=\frac{z}{\sigma^{2}} e^{-\frac{\left(z^{2}+v^{2}\right)}{2 \sigma^{2}}} I_{0}\left(\frac{z v}{\sigma^{2}}\right), \quad z \geq 0
$$

where $I_{0}$ is the modified Bessel function of the first kind with order zero. From (29), we can observe that the LoS phase $\theta$ does not affect the distribution. Adopting the notation for the channel $h_{m}$ gives that $2 \sigma^{2}=\beta=\frac{1}{M} \operatorname{tr}(\mathbf{R})$ is the average channel gain of NLoS multipath components at the antenna $m$ and $v^{2}=\bar{h}_{m}^{2}$ is the power in the LoS component. The total average received power in the Rician fading can be calculated by

$$
P=\int_{0}^{\infty} z^{2} p_{Z}(z) d z=2 \sigma^{2}+s^{2} .
$$

and substituting $z=\left|h_{m}\right|$ gives $P=\beta+\bar{h}_{m}^{2}$. The ratio of the power in the LoS component to the power in the NLoS component is denoted by the Rician factor $\kappa$. It is equal to $\kappa=\frac{\bar{h}_{m}^{2}}{\beta}$ and shows the level of fading. The portion of the power that the LoS path contains is $\bar{h}_{m}^{2}=\frac{\kappa P}{\kappa+1}$ and the NLoS part has the power $\beta=\frac{P}{\kappa+1}$. Note that for $\kappa=0$, we have Rayleigh fading since the there is no power in the LoS path. Conversely, if $\kappa \rightarrow \infty$ we have no multipath meaning that there is only the LoS component. It shows that a small $\kappa$ implies severe fading whereas a larger $\kappa$ implies a milder fading.

To simplify the analysis, the LoS phase shift $\theta$ and the spatial correlation are often neglected. The LoS phase is typically assumed to be fixed or can be tracked perfectly. As discussed above, neglecting the phase shift does not change the distribution of the envelope. However, the resulting model becomes physically less accurate. In Paper A, we considered a co-located massive MIMO system where the LoS phase is identical for all base station antennas and may therefore be accurately tracked in practice. In co-located MIMO systems, the LoS phase can be estimated by employing classical algorithms such as estimation of signal parameters via rotational invariance techniques (ESPRIT) [50] and multiple signal classification (MUSIC) [51]. However, these methods either require multiple antennas and/or long sample sequences (snapshots). In Paper B, we studied a cell-free massive MIMO system where each access point has a single antenna. Consequently, it is harder to estimate the LoS phase. In a low mobility scenario in which the access points are equipped 
with multiple antennas, the phase-shifts can be estimated quite well, but the estimates will anyway be imperfect since the estimation resources are practically limited. In Paper B, to see the effect of the phase shift on system performance, we studied two cases where the phase is either known or completely unknown.

\subsubsection{Impact of LoS path and Spatial Correlation in Massive MIMO}

In this section, we will demonstrate the basic impacts of LoS path and spatial correlation in massive MIMO. In early single-user MIMO research, spatial correlation is viewed as a detrimental effect $[52,53]$. However, it may not be the case in the multiuser massive MIMO communications. The spatial correlation can be both beneficial and detrimental depending on the relation between the dominant eigenvectors of the active users. Similarly, in some cases such as when the AoAs of two users are not distinct enough to be resolved by the base station, the existence of an LoS paths can be detrimental as well [54]. However, any standard scheduling scheme can compensate for this degradation by simply dropping these users. To understand the effects of the spatial correlation and the LoS path, we first visit two key properties of massive MIMO: channel hardening and favorable propagation.

A propagation channel $\mathbf{h}$ provides channel hardening if

$$
\frac{\|\mathbf{h}\|^{2}}{\mathbb{E}\left\{\mid \mathbf{h} \|^{2}\right\}} \rightarrow 1
$$

as $M \rightarrow \infty$. It implies that the value of instantaneous channel gain $\|\mathbf{h}\|^{2}$ is close to its mean value $\mathbb{E}\left\{\|\mathbf{h}\|^{2}\right\}$ when the number of antennas is quite large. In other words, as more antennas are added the channel variations reduce. However, it does not mean that $\|\mathbf{h}\|^{2} \rightarrow \mathbb{E}\left\{\mid \mathbf{h} \|^{2}\right\}$ since both terms generally diverge as $M \rightarrow \infty$. We can quantify how close we are to the asymptotic channel hardening when we have practical number of antennas by calculating $\operatorname{Var}\left\{\frac{\|\mathbf{h}\|^{2}}{\mathbb{E}\left\{\mid \mathbf{h} \|^{2}\right\}}\right\}$. If the variance goes to zero, we obtain convergence in probability. Using Lemma 5 from Paper A, for spatially correlated Rician fading channels, we can obtain

$$
\begin{aligned}
\operatorname{Var}\left\{\frac{\|\mathbf{h}\|^{2}}{\mathbb{E}\left\{\mid \mathbf{h} \|^{2}\right\}}\right\} & =\mathbb{E}\left\{\left(\frac{\|\mathbf{h}\|^{2}}{\mathbb{E}\left\{\|\mathbf{h}\|^{2}\right\}}\right)^{2}\right\}-\left(\mathbb{E}\left\{\frac{\|\mathbf{h}\|^{2}}{\mathbb{E}\left\{\|\mathbf{h}\|^{2}\right\}}\right\}\right)^{2} \\
& =\frac{\mathbb{E}\left\{\|\mathbf{h}\|^{4}\right\}-\left(\mathbb{E}\left\{\|\mathbf{h}\|^{2}\right\}\right)^{2}}{\left(\mathbb{E}\left\{\|\mathbf{h}\|^{2}\right\}\right)^{2}}=\frac{\operatorname{tr}\left(\mathbf{R}^{2}\right)+2 \operatorname{Re}\left\{\overline{\mathbf{h}}^{H} \mathbf{R} \overline{\mathbf{h}}\right\}}{\left(\operatorname{tr}(\mathbf{R})+\|\overline{\mathbf{h}}\|^{2}\right)^{2}}
\end{aligned}
$$

and it should be close to zero if channel hardening is to be observed. In the special case of i.i.d. Rayleigh fading, $\mathbf{R}=\beta \mathbf{I}_{M}$ (32) becomes $\frac{\operatorname{tr}\left(\mathbf{R}^{2}\right)}{\operatorname{tr}(\mathbf{R})^{2}}=\frac{1}{M}$. Similarly, for 
i.i.d. Rician fading with $\|\overline{\mathbf{h}}\|^{2}=\beta^{\text {los }} M$, the variance in (32) is

$$
\frac{1}{M} \frac{\beta^{2}+2 \beta \beta^{\text {los }}}{\beta^{2}+2 \beta \beta^{\text {los }}+\left(\beta^{\text {los }}\right)^{2}}=\frac{1}{M} \frac{2 \kappa+1}{\kappa^{2}+2 \kappa+1}
$$

using the definition of Rician factor $\kappa=\frac{\beta^{\text {los }}}{\beta}$. Figure 5 compares the variance of channel hardening defined in (32) as a function of the number of antennas for different channel models. Here the covariance matrices are normalized such that $\operatorname{tr}(\mathbf{R})=M$ for both correlated and i.i.d. fading. Likewise, the LoS path is normalized as $\|\overline{\mathbf{h}}\|^{2}=M$. Note that this normalization gives a Rician factor $\kappa=1$. As can be observed from the figure, the existence of LoS improves the channel hardening. It should be noted that $\kappa=1$ implies rather severe fading and as $\kappa$ increases the gap between Rayleigh and Rician models will increase as well. In addition, we can observe that spatial correlation reduces the channel hardening since the eigenvalue variations in $\mathbf{R}$ increase. To summarize, spatial correlation reduces and the LoS path increases the level of channel hardening.

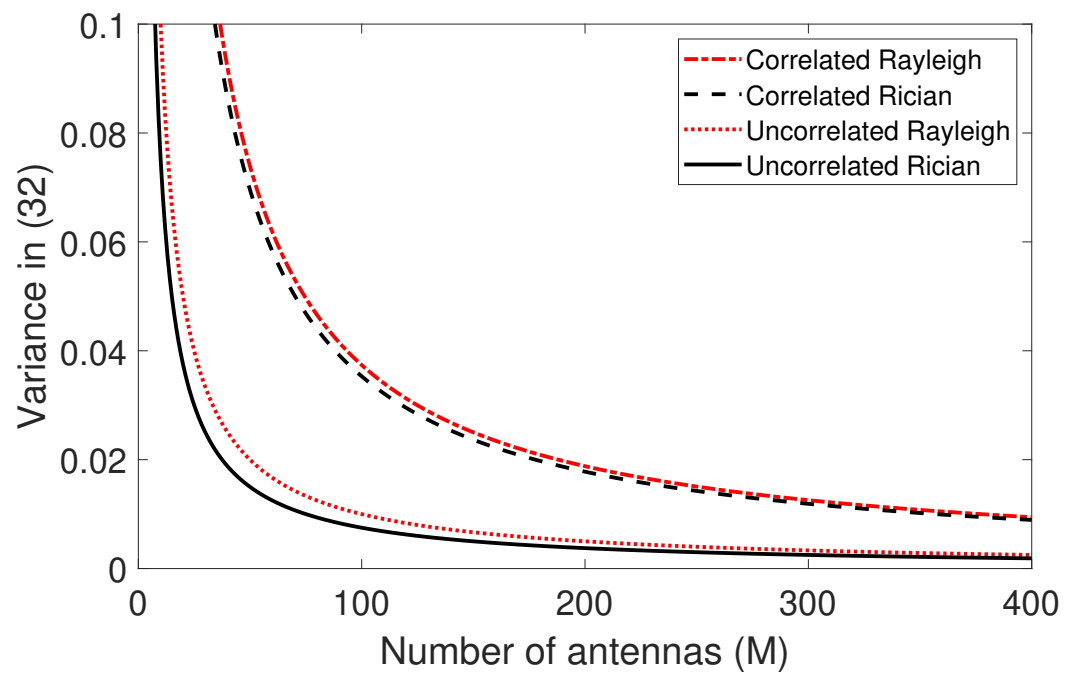

Figure 5: Variance of channel hardening defined in (32) as a function of the number of antennas. Correlated fading is generated using local scattering model with the nominal angle $\varphi=30^{\circ}$, ASD $10^{\circ}$ and Gaussian angular distribution.

Another key property of massive MIMO, favorable propagation, implies that the propagation channel responses from the base station to two distinct users point in sufficiently different directions. In other words, the favorable propagation makes the directions of two users channels asymptotically orthogonal. This property helps the base station to mitigate interference between the users, without losing much in 
desired signal power. Assume that the channels of the users are denoted by $\mathbf{h}_{1}$ and $\mathbf{h}_{2}$, respectively. The pair of channels $\mathbf{h}_{1}$ and $\mathbf{h}_{2}$ provide asymptotically favorable propagation if

$$
\frac{\mathbf{h}_{1}^{H} \mathbf{h}_{2}}{\sqrt{\mathbb{E}\left\{\left\|\mathbf{h}_{1}\right\|^{2}\right\} \mathbb{E}\left\{\left\|\mathbf{h}_{2}\right\|^{2}\right\}}} \rightarrow 0
$$

as $M \rightarrow \infty$. Similar to channel hardening discussion, we can quantify how close we are to asymptotic favorable propagation with practical number of antennas by calculating $\operatorname{Var}\left\{\frac{\mathbf{h}_{1}^{H} \mathbf{h}_{2}}{\sqrt{\mathbb{E}\left\{\left\|\mathbf{h}_{1}\right\|^{2}\right\} \mathbb{E}\left\{\mid \mathbf{h}_{2} \|^{2}\right\}}}\right\}$. Using Lemma 4 from Paper A, for spatially correlated Rician fading channels, we can calculate

$$
\begin{aligned}
& \operatorname{Var}\left\{\frac{\mathbf{h}_{1}^{H} \mathbf{h}_{2}}{\left.\sqrt{\mathbb{E}\left\{\left\|\mathbf{h}_{1}\right\|^{2}\right\} \mathbb{E}\left\{\left\|\mathbf{h}_{2}\right\|^{2}\right\}}\right\}}\right. \\
& =\frac{\operatorname{tr}\left(\mathbf{R}_{1} \mathbf{R}_{2}\right)+\operatorname{Re}\left\{\overline{\mathbf{h}}_{2}^{H} \mathbf{R}_{1} \overline{\mathbf{h}}_{2}\right\}+\operatorname{Re}\left\{\overline{\mathbf{h}}_{1}^{H} \mathbf{R}_{2} \overline{\mathbf{h}}_{1}\right\}+\left|\overline{\mathbf{h}}_{1}^{H} \overline{\mathbf{h}}_{2}\right|^{2}}{\left(\operatorname{tr}\left(\mathbf{R}_{1}\right)+\left\|\overline{\mathbf{h}}_{1}\right\|^{2}\right)\left(\operatorname{tr}\left(\mathbf{R}_{2}\right)+\left\|\overline{\mathbf{h}}_{2}\right\|^{2}\right)}
\end{aligned}
$$

and it should be close to zero if favorable propagation is to be observed.

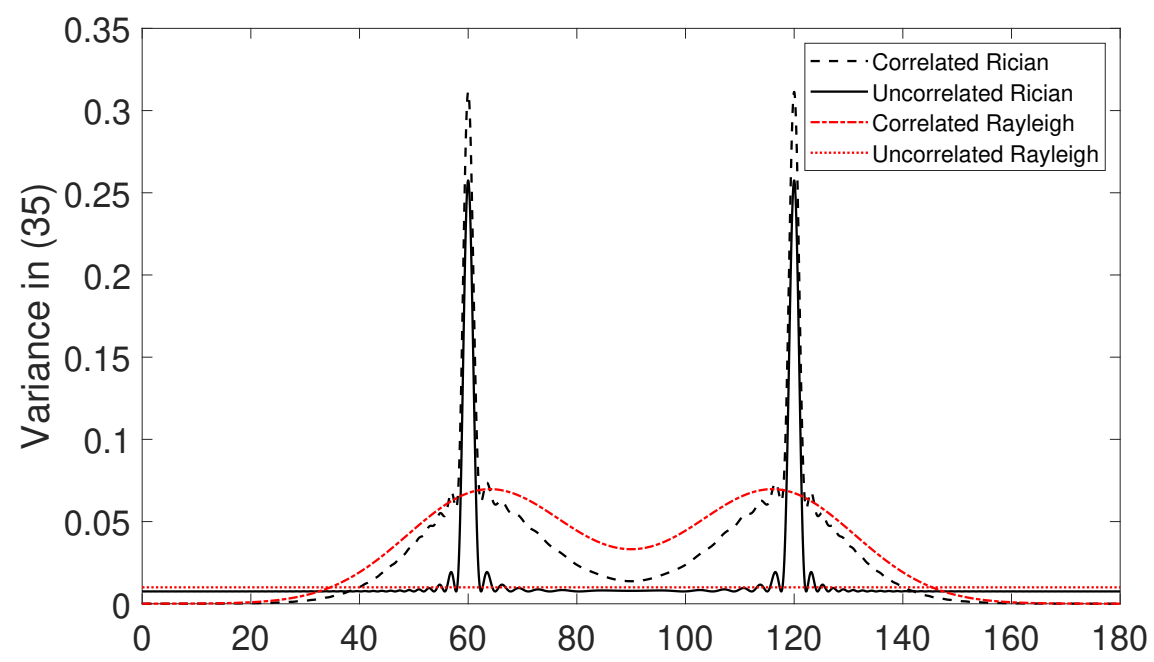

Figure 6: Variance of favorable propagation for $M=100$ and ASD $10^{\circ}$ (with Gaussian angular distribution) if a correlated channel model is used. The user 1 has a nominal angle of $60^{\circ}$, while the angle of the interfering user (the user 2 ) is varied $\left[0^{\circ}, 180^{\circ}\right]$.

Figure 6 compares the levels of favorable propagation for different channel models. It shows that the effect of the LoS path is quite prominent if the users have 
similar nominal angles. This is also evident from (9) since the base station is not able to distinguish the channels of users with similar angles. From Figure 2, we can deduce that these peaks will get narrower as the number of antennas increase since the resolution of ULA will increase. Moreover, we observe that spatial correlation increases the levels of favorable propagation if the covariance matrices of the users are different enough. 


\title{
Chapter 2
}

\section{Contributions of the Thesis}

This thesis focuses on analysis of cellular and cell-free massive MIMO with Rician fading. In this section, we provide the publication information for the papers that are included in this thesis and further list other publications that are not included since they are preliminary versions of the included papers or not within the main scope of this thesis.

\subsection{Papers Included in the Thesis}

\section{Paper A: Massive MIMO With Spatially Correlated Rician Fading Channels}

Authored by: Özgecan Özdogan, Emil Björnson, and Erik G. Larsson

Published in: IEEE Transactions on Communications, vol. 67, no. 5, pp. 3234-3250, May 2019.

\begin{abstract}
This paper considers multi-cell massive multiple-input multiple-output systems, where the channels are spatially correlated Rician fading. The channel model is composed of a deterministic line-of-sight path and a stochastic non-line-ofsight component describing a practical spatially correlated multipath environment. We derive the statistical properties of the minimum mean squared error (MMSE), element-wise MMSE, and least-square channel estimates for this model. Using these estimates for maximum ratio combining and precoding, rigorous closed-form uplink (UL) and downlink (DL) achievable spectral efficiency (SE) expressions are derived and analyzed. The asymptotic SE behavior, when using the different channel estimators, are also analyzed. The numerical results show that the SE is higher when using the MMSE estimator than that of the other estimators, and the performance gap increases with the number of antennas.
\end{abstract}




\title{
Paper B: Performance of Cell-Free Massive MIMO With Rician Fading and Phase Shifts
}

Authored by: Özgecan Özdogan, Emil Björnson, and Jiayi Zhang

Published in: IEEE Transactions on Wireless Communications, vol. 18, no. 11, pp. 5299-5315, Nov. 2019.

\begin{abstract}
In this paper, we study the uplink (UL) and downlink (DL) spectral efficiency (SE) of a cell-free massive multiple-input-multiple-output (MIMO) system over Rician fading channels. The phase of the line-of-sight (LoS) path is modeled as a uniformly distributed random variable to take the phase-shifts due to mobility and phase noise into account. Considering the availability of prior information at the access points (APs), the phase-aware minimum mean square error (MMSE), non-aware linear MMSE (LMMSE), and least-square (LS) estimators are derived. The MMSE estimator requires perfectly estimated phase knowledge whereas the LMMSE and LS are derived without it. In the UL, a two-layer decoding method is investigated in order to mitigate both coherent and non-coherent interference. Closedform UL SE expressions with phase-aware MMSE, LMMSE, and LS estimators are derived for maximum-ratio (MR) combining in the first layer and optimal large-scale fading decoding (LSFD) in the second layer. In the DL, two different transmission modes are studied: coherent and non-coherent. Closed-form DL SE expressions for both transmission modes with MR precoding are derived for the three estimators. Numerical results show that the LSFD improves the UL SE performance and coherent transmission mode performs much better than non-coherent transmission in the DL. Besides, the performance loss due to the lack of phase information depends on the pilot length and it is small when the pilot contamination is low.
\end{abstract}




\subsection{Papers Not Included in the Thesis}

The following publications contain the works that are done by the author but are not included in the thesis since they were either earlier versions of the journals or not fixed well within the main scope of the thesis.

\section{Uplink Spectral Efficiency of Massive MIMO with Spatially Correlated Rician Fading}

Authored by: Özgecan Özdogan, Emil Björnson, and Erik G. Larsson

Published in: 2018 IEEE 19th International Workshop on Signal Processing Advances in Wireless Communications (SPAWC), Kalamata, 2018, pp. 1-5.

This paper contains preliminary results of Paper A.

Abstract: This paper considers the uplink (UL) of a multicell Massive MIMO (multiple-input multiple-output) system with spatially correlated Rician fading channels. The channel model is composed of a deterministic line-of-sight (LoS) path and a stochastic non-line-of-sight (NLoS) component describing a spatially correlated multipath environment. We derive the statistical properties of the minimum mean squared error (MMSE) and least-square (LS) channel estimates for this model. Using these estimates for maximum ratio (MR) combining, rigorous closed-form UL spectral efficiency (SE) expressions are derived. Numerical results show that the SE is higher when using the MMSE estimator than the LS estimator, and the performance gap increases with the number of antennas. Moreover, Rician fading provides higher achievable SEs than Rayleigh fading since the LoS path improves the sum SE.

\section{Cell-Free Massive MIMO with Rician Fading: Estimation Schemes and Spectral Efficiency}

Authored by: Özgecan Özdogan, Emil Björnson, and Jiayi Zhang

Published in: 2018 52nd Asilomar Conference on Signals, Systems, and Computers, Pacific Grove, CA, USA, 2018, pp. 975-979.

Abstract: As the cell sizes in cellular networks shrink, the inter-cell interference becomes more of an issue. Instead of operating each cell autonomously, we can connect all the access points (APs) together to form a cell-free massive MIMO (multiple-input multiple-output) system that can alleviate interference by spatial processing. Previous studies have focused on Rayleigh fading channels, but in densely deployed systems, it is likely that some of the users will have line-of-sight (LoS) propagation to some of the APs. In this paper, we model this by arbitrarily distributed 
Rician fading channels. Two types of channel estimators are considered: a classical least-square (LS) estimator and a Bayesian minimum mean square error (MMSE) estimator. We derive closed-form spectral efficiency (SE) expressions for the uplink (UL) and downlink (DL) when using each of these estimators for maximum ratio (MR) processing. The performance difference is evaluated numerically to figure out under which conditions it is beneficial to know the channel statistics when estimating a channel.

\section{Downlink Performance of Cell-Free Massive MIMO with Rician Fading and Phase Shifts}

Authored by: Özgecan Özdogan, Emil Björnson, and Jiayi Zhang

Published in: 2019 IEEE 20th International Workshop on Signal Processing Advances in Wireless Communications (SPAWC), Cannes, France, 2019, pp. 1-5.

This paper contains preliminary results of Paper B.

Abstract: In this paper, we study the downlink (DL) spectral efficiency (SE) of a cell-free massive multiple-input-multiple-output (MIMO) system with Rician fading channels. The phase of the line-of-sight (LoS) path is modeled as a uniformly distributed random variable to take the phase-shifts due to mobility and phase noise into account. Considering the availability of prior information at the access points (APs), the phase-aware minimum mean square error (MMSE) and non-aware linear MMSE (LMMSE) estimators are derived. The MMSE estimator requires perfectly estimated phase knowledge whereas the LMMSE is derived without it. Besides, two different transmission modes are studied: coherent and non-coherent. Closed-form DL SE expressions for both coherent and non-coherent transmission with maximumratio (MR) precoding are derived for the two estimators. Numerical results show that the performance loss due to the lack of phase information is small and coherent transmission mode performs much better than non-coherent transmission.

\section{Intelligent Reflecting Surface vs. Decode-and-Forward: How Large Surfaces Are Needed to Beat Relaying?}

Authored by: Emil Björnson, Özgecan Özdogan, and Erik G. Larsson Published in: IEEE Wireless Communications Letters

Abstract: The rate and energy efficiency of wireless channels can be improved by
deploying software-controlled metasurfaces to reflect signals from the source to
destination, especially when the direct path is weak. While previous works mainly
optimized the reflections, this letter compares the new technology with classic
decode-and-forward (DF) relaying. The main observation is that very high rates 
and/or large metasurfaces are needed to outperform DF relaying, both in terms of minimizing the total transmit power and maximizing the energy efficiency, which also includes the dissipation in the transceiver hardware.

\title{
Intelligent Reflecting Surfaces: Physics, Propagation, and Pathloss Modeling
}

Authored by: Özgecan Özdogan, Emil Björnson, and Erik G. Larsson

Published in: IEEE Wireless Communications Letters

\begin{abstract}
Intelligent reflecting surfaces can improve the communication between a source and a destination. The surface contains metamaterial that is configured to "reflect" the incident wave from the source towards the destination. Two incompatible pathloss models have been used in prior work. In this letter, we derive the far-field pathloss using physical optics techniques and explain why the surface consists of many elements that individually act as diffuse scatterers but can jointly beamform the signal in a desired direction with a certain beamwidth. We disprove one of the previously conjectured pathloss models.
\end{abstract}

\section{Using Intelligent Reflecting Surfaces for Rank Improvement in MIMO Communications}

Authored by: Özgecan Özdogan, Emil Björnson, and Erik G. Larsson

Accepted to: 45th International Conference on Acoustics, Speech, and Signal Processing (ICASSP) 2020

Abstract: An intelligent reflecting surface (IRS), consisting of reconfigurable metamaterials, can be used to partially control the radio environment and thereby bring new features to wireless communications. Previous works on IRS have particularly studied the range extension use case and under what circumstances the new technology can beat relays. In this paper, we study another use case that might have a larger impact on the channel capacity: rank improvement. One of the classical bottlenecks of point-to-point MIMO communications is that the capacity gains provided by spatial multiplexing are only large at high SNR, and high SNR channels are mainly appearing in line-of-sight (LoS) scenarios where the channel matrix has low rank and therefore does not support spatial multiplexing. We demonstrate how an IRS can be used and optimized in such scenarios to increase the rank of the channel matrix, leading to substantial capacity gains. 


\section{Bibliography}

[1] M. Cooper, “The myth of spectrum scarcity,” DYNA llc, Tech. Rep., Mar. 2010.

[2] H. H. Beverage and H. O. Peterson, "Diversity receiving system of R.C.A. communications, inc., for radiotelegraphy," Proceedings of the Institute of Radio Engineers, vol. 19, no. 4, pp. 529-561, April 1931.

[3] Cisco, "Visual networking index: Global mobile data traffic forecast update, 2016 - 2021," Tech. Rep., Feb. 2017.

[4] T. L. Marzetta, "Noncooperative cellular wireless with unlimited numbers of base station antennas," IEEE Transactions on Wireless Communications, vol. 9, no. 11, pp. 3590-3600, November 2010.

[5] T. L. Marzetta, E. G. Larsson, H. Yang, and H. Q. Ngo, "Fundamentals of massive MIMO," Cambridge University Press, 2016.

[6] E. Björnson, J. Hoydis, and L. Sanguinetti, "Massive MIMO networks: Spectral, energy, and hardware efficiency," Foundations and Trends ${ }^{\circledR}$ in Signal Processing, vol. 11, no. 3-4, pp. 154-655, 2017.

[7] J. Hoydis, S. ten Brink, and M. Debbah, "Massive MIMO in the UL/DL of cellular networks: How many antennas do we need?" IEEE Journal on Selected Areas in Communications, vol. 31, no. 2, pp. 160-171, February 2013.

[8] H. Yang and T. L. Marzetta, "Massive MIMO with max-min power control in line-of-sight propagation environment," IEEE Transactions on Communications, vol. 65, no. 11, pp. 4685-4693, Nov 2017.

[9] C. Chen, A. P. Guevara, and S. Pollin, "Scaling up distributed massive MIMO: Why and how," in 201751 st Asilomar Conference on Signals, Systems, and Computers, Oct 2017, pp. 271-276.

[10] C. Chen, S. Blandino, A. Gaber, C. Desset, A. Bourdoux, L. Van der Perre, and S. Pollin, "Distributed massive MIMO: A diversity combining method 
for TDD reciprocity calibration," in GLOBECOM 2017 - 2017 IEEE Global Communications Conference, Dec 2017, pp. 1-7.

[11] K. T. Truong and R. W. Heath, "The viability of distributed antennas for massive MIMO systems," in 2013 Asilomar Conference on Signals, Systems and Computers, Nov 2013, pp. 1318-1323.

[12] H. Q. Ngo, A. Ashikhmin, H. Yang, E. G. Larsson, and T. L. Marzetta, "Cellfree massive MIMO versus small cells," IEEE Transactions on Wireless Communications, vol. 16, no. 3, pp. 1834-1850, March 2017.

[13] E. Nayebi, A. Ashikhmin, T. L. Marzetta, H. Yang, and B. D. Rao, "Precoding and power optimization in cell-free massive MIMO systems," IEEE Transactions on Wireless Communications, vol. 16, no. 7, pp. 4445-4459, July 2017.

[14] G. Interdonato, E. Björnson, H. N. Quoc, P. Frenger, and E. G. Larsson, "Ubiquitous cell-free massive MIMO communications," EURASIP Journal on Wireless Communications and Networking, vol. 2019, no. 1, p. 197, Aug 2019.

[15] J. Zhang, S. Chen, Y. Lin, J. Zheng, B. Ai, and L. Hanzo, "Cell-free massive MIMO: A new next-generation paradigm," IEEE Access, vol. 7, pp. 99 878-99 888, 2019.

[16] D. Gesbert, S. Hanly, H. Huang, S. Shamai Shitz, O. Simeone, and W. Yu, "Multi-cell MIMO cooperative networks: A new look at interference," IEEE Journal on Selected Areas in Communications, vol. 28, no. 9, pp. 1380-1408, December 2010.

[17] S. Venkatesan, A. Lozano, and R. Valenzuela, "Network MIMO: Overcoming intercell interference in indoor wireless systems," in 2007 Conference Record of the Forty-First Asilomar Conference on Signals, Systems and Computers, Nov 2007, pp. 83-87.

[18] G. J. Foschini, K. Karakayali, and R. A. Valenzuela, "Coordinating multiple antenna cellular networks to achieve enormous spectral efficiency," IEE Proceedings - Communications, vol. 153, no. 4, pp. 548-555, August 2006.

[19] R. Irmer, H. Droste, P. Marsch, M. Grieger, G. Fettweis, S. Brueck, H. Mayer, L. Thiele, and V. Jungnickel, "Coordinated Multipoint: Concepts, performance, and field trial results," IEEE Communications Magazine, vol. 49, no. 2, pp. 102-111, February 2011. 
[20] E. Björnson, R. Zakhour, D. Gesbert, and B. Ottersten, "Cooperative Multicell Precoding: Rate region characterization and distributed strategies with instantaneous and statistical CSI," IEEE Transactions on Signal Processing, vol. 58, no. 8, pp. 4298-4310, Aug 2010.

[21] R. W. Heath Jr. and A. Lozano, "Foundations of MIMO communication," Cambridge University Press, 2018.

[22] S. Hur, S. Baek, B. Kim, J. Park, A. F. Molisch, K. Haneda, and M. Peter, "28 $\mathrm{GHz}$ channel modeling using 3D ray-tracing in urban environments," in 2015 9th European Conference on Antennas and Propagation (EuCAP), April 2015, pp. 1-5.

[23] B. Guo, Y. Wu, M. Yang, and J. Li, "28 GHz millimeter wave propagation models based on ray-tracing in urban scenario," in 2015 IEEE 26th Annual International Symposium on Personal, Indoor, and Mobile Radio Communications (PIMRC), Aug 2015, pp. 2209-2213.

[24] M. Jacob, S. Priebe, A. Maltsev, A. Lomayev, V. Erceg, and T. Kürner, "A ray tracing based stochastic human blockage model for the IEEE $802.11 \mathrm{ad} 60 \mathrm{GHz}$ channel model," in Proceedings of the 5th European Conference on Antennas and Propagation (EUCAP), April 2011, pp. 3084-3088.

[25] D. Tse and P. Viswanath, "Fundamentals of wireless communication," Cambridge University Press, 2005.

[26] A. Goldsmith, "Wireless communications," Cambridge University Press, 2005.

[27] A. Papoulis and S. U. Pillai, "Probability, random variables, and stochastic processes," McGraw Hill, 2002.

[28] M. Slack, "The probability distributions of sinusoidal oscillations combined in random phase," Journal of the Institution of Electrical Engineers - Part III: Radio and Communication Engineering, vol. 93, no. 22, pp. 76-86, March 1946.

[29] W. R. Bennet, "Distribution of the sum of randomly phased components," Quarterly of Applied Mathematics, vol. 5, no. 4, p. 385-393, 1948.

[30] S. Blandino, C. Desset, G. Mangraviti, A. Bourdoux, and S. Pollin, "Phasenoise mitigation at $60 \mathrm{GHz}$ with a novel hybrid MIMO architecture," in Proceedings of the 2nd ACM Workshop on Millimeter Wave Networks and Sensing Systems, ser. mmNets '18, New York, NY, USA, 2018, p. 39-44. 
[31] R. H. Clarke, "A Statistical Theory of Mobile-Radio Reception," Bell System Technical Journal, vol. 47, no. 6, pp. 957-1000, 81968.

[32] W. Jakes and D. C. Cox, "Microwave mobile communications," Wiley-IEEE Press, 1994.

[33] X. Gao, O. Edfors, F. Tufvesson, and E. G. Larsson, "Massive MIMO in real propagation environments: Do all antennas contribute equally?" IEEE Transactions on Communications, vol. 63, no. 11, pp. 3917-3928, Nov 2015.

[34] J. P. Kermoal, L. Schumacher, K. I. Pedersen, P. E. Mogensen, and F. Frederiksen, "A stochastic MIMO radio channel model with experimental validation," IEEE Journal on Selected Areas in Communications, vol. 20, no. 6, pp. 1211-1226, Aug 2002.

[35] X. Gao, O. Edfors, F. Rusek, and F. Tufvesson, "Massive MIMO performance evaluation based on measured propagation data," IEEE Transactions on Wireless Communications, vol. 14, no. 7, pp. 3899-3911, July 2015.

[36] A. Adhikary, J. Nam, J.-Y. Ahn, and G. Caire, "Joint spatial division and multiplexing-The large-scale array regime," IEEE Transactions on Information Theory, vol. 59, no. 10, pp. 6441-6463, 2013.

[37] J. Salz and J. H. Winters, "Effect of fading correlation on adaptive arrays in digital mobile radio," IEEE Transactions on Vehicular Technology, vol. 43, no. 4, pp. 1049-1057, 1994.

[38] D. S. Shiu, G. J. Foschini, M. J. Gans, and J. M. Kahn, "Fading correlation and its effect on the capacity of multielement antenna systems," IEEE Transactions on Communications, vol. 48, no. 3, pp. 502-513, March 2000.

[39] H. Yin, D. Gesbert, M. Filippou, and Y. Liu, "A coordinated approach to channel estimation in large-scale multiple-antenna systems," IEEE Journal on Selected Areas in Communications, vol. 31, no. 2, pp. 264-273, February 2013.

[40] A. F. Molisch, "Wireless communications," John Wiley \& Sons, 2007.

[41] Z. Jiang, A. F. Molisch, G. Caire, and Z. Niu, "Achievable rates of FDD massive MIMO systems with spatial channel correlation," IEEE Transactions on Wireless Communications, vol. 14, no. 5, pp. 2868-2882, May 2015.

[42] F. Adachi, M. T. Feeney, J. D. Parsons, and A. G. Williamson, "Crosscorrelation between the envelopes of $900 \mathrm{MHz}$ signals received at a mobile radio base 
station site," IEE Proc. F - Commun., Radar and Signal Process., vol. 133, no. 6, pp. 506-512, 1986.

[43] P. Zetterberg and B. Ottersten, "The spectrum efficiency of a base station antenna array system for spatially selective transmission," IEEE Transactions on Vehicular Technology, vol. 44, no. 3, pp. 651-660, 1995.

[44] J. Salz and J. H. Winters, "Effect of fading correlation on adaptive arrays in digital mobile radio," IEEE Transactions on Vehicular Technology, vol. 43, no. 4, pp. 1049-1057, Nov 1994.

[45] R. Stridh, "Smart antennas in wireless networks: System issues and performancs limits," Ph.D. dissertation, KTH Royal Institute of Technology, 2003.

[46] R. Kennedy, "Fading dispersive communication channels," Wiley-Interscience, 1969.

[47] R. M. Gray, "Toeplitz and circulant matrices: A review," Foundations and Trends ${ }^{\circledR}$ in Communications and Information Theory, vol. 2, no. 3, pp. 155-239, 2006. [Online]. Available: http://dx.doi.org/10.1561/0100000006

[48] 3rd Generation Partnership Project, "Technical Specification Group Radio Access Network; Spatial channel model for Multiple Input Multiple Output (MIMO) simulations", Mar. 2017, 3GPP TR 25.996 V14.0.0.

[49] S. O. Rice, "Mathematical analysis of random noise," The Bell System Technical Journal, vol. 23, no. 3, pp. 282-332, July 1944.

[50] A. Paulraj, R. Roy, and T. Kailath, "Estimation of signal parameters via rotational invariance techniques- ESPRIT," in Nineteeth Asilomar Conference on Circuits, Systems and Computers, 1985., Nov 1985, pp. 83-89.

[51] R. O. Schmidt, "A signal subspace approach to multiple emitter location and spectral estimation," Ph.D. dissertation, 1981.

[52] S. L. Loyka, "Channel capacity of MIMO architecture using the exponential correlation matrix," IEEE Communications Letters, vol. 5, no. 9, pp. 369-371, Sep. 2001.

[53] A. J. Paulraj and C. B. Papadias, "Space-time processing for wireless communications," IEEE Signal Processing Magazine, vol. 14, no. 6, pp. 49-83, Nov 1997. 
[54] M. Matthaiou, P. J. Smith, H. Q. Ngo, and H. Tataria, "Does massive MIMO fail in Ricean channels?" IEEE Wireless Communications Letters, vol. 8, no. 1, pp. 61-64, Feb 2019. 


\section{Included Papers}





\section{Papers}

The papers associated with this thesis have been removed for copyright reasons. For more details about these see:

http://urn.kb.se/resolve?urn=urn:nbn:se:liu:diva-163688 


\section{Other Recently Published Theses From \\ The Division of Communication Systems \\ Department of Electrical Engineering (ISY) \\ Linköping University, Sweden}

Ema Becirovic, On Massive MIMO for Massive Machine-Type Communications, Linköping Studies in Science and Technology. Licentiate Thesis, No. 1868, 2020.

Daniel Verenzuela, Exploring Alternative Massive MIMO Designs: Superimposed Pilots and Mixed-ADCs, Linköping Studies in Science and Technology. Dissertations, No. 2041, 2020.

Trịnh Văn Chiến, Spatial Resource Allocation in Massive MIMO Communication: From Cellular to Cell-Free, Linköping Studies in Science and Technology. Dissertations, No. 2036, 2020.

Amin Ghazanfari, Power Control for Multi-Cell Massive MIMO, Linköping Studies in Science and Technology. Licentiate Thesis, No. 1852, 2019.

Giovanni Interdonato, Signal Processing Aspects of Cell-Free Massive MIMO, Linköping Studies in Science and Technology. Licentiate Thesis, No. 1817, 2018.

Marcus Karlsson, Blind Massive MIMO Base Stations: Downlink Transmission and Jamming, Linköping Studies in Science and Technology. Dissertations, No. 1950, 2018.

Victor Hei Cheng, Optimizing Massive MIMO: Precoder Design and Power Allocation, Linköping Studies in Science and Technology. Dissertations, No. 1929, 2018.

Christopher Mollén, High-End Performance with Low-End Hardware: Analysis of Massive MIMO Base Station Transceivers, Linköping Studies in Science and Technology. Dissertations, No. 1896, 2017.

Antonios Pitarokoilis, Phase Noise and Wideband Transmission in Massive MIMO, Linköping Studies in Science and Technology. Dissertations, No. 1756, 2016.

Anu Kalidas M. Pillai, Signal Reconstruction Algorithms for Time-Interleaved ADCs, Linköping Studies in Science and Technology. Dissertations, No. 1672, 2015.

Ngô Quốc Hiển, Massive MIMO: Fundamentals and System Designs, Linköping Studies in Science and Technology. Dissertations, No. 1642, 2015.

Mirsad Čirkić, Efficient MIMO Detection Methods, Linköping Studies in Science and Technology. Dissertations, No. 1570, 2014.

Reza Moosavi, Improving the Efficiency of Control Signaling in Wireless Multiple Access Systems, Linköping Studies in Science and Technology. Dissertations, No. 1556, 2014. 


\section{FACULTY OF SCIENCE AND ENGINEERING}

Linköping Studies in Science and Technology,

Licentiate Thesis No. 1870, 2020

Division of Communication Systems

Department Of Electrical Engineering (ISY)

Linköping University

SE-581 83 Linköping, Sweden

www.liu.se 